\title{
Review Article \\ On the Definition of Energy for a Continuum, Its Conservation Laws, and the Energy-Momentum Tensor
}

\author{
Mayeul Arminjon \\ Laboratory "Soils, Solids, Structures, Risks", 3SR, Grenoble Alpes University and CNRS, Domaine Universitaire, \\ BP 53, 38041 Grenoble Cedex 9, France \\ Correspondence should be addressed to Mayeul Arminjon; mayeul.arminjon@3sr-grenoble.fr
}

Received 11 May 2016; Accepted 15 June 2016

Academic Editor: Manuel De León

Copyright (C) 2016 Mayeul Arminjon. This is an open access article distributed under the Creative Commons Attribution License, which permits unrestricted use, distribution, and reproduction in any medium, provided the original work is properly cited.

We review the energy concept in the case of a continuum or a system of fields. First, we analyze the emergence of a true local conservation equation for the energy of a continuous medium, taking the example of an isentropic continuum in Newtonian gravity. Next, we consider a continuum or a system of fields in special relativity: we recall that the conservation of the energy-momentum tensor contains two local conservation equations of the same kind as before. We show that both of these equations depend on the reference frame and that, however, they can be given a rigorous meaning. Then, we review the definitions of the canonical and Hilbert energy-momentum tensors from a Lagrangian through the principle of stationary action in general space-time. Using relatively elementary mathematics, we prove precise results regarding the definition of the Hilbert tensor field, its uniqueness, and its tensoriality. We recall the meaning of its covariant conservation equation. We end with a proof of uniqueness of the energy density and flux, when both depend polynomially on the fields.

\section{Introduction and Summary}

The subject of this paper is wide and there is a huge literature about it. The aim of the paper is to give a unified exposition of what, in this author's view, are the main aspects of the subject, in a relatively short space, while, nevertheless, emphasizing or precising some not widely appreciated facts and providing strict proofs of some less obvious matters (mainly the Hilbert energy-momentum tensor), using not too sophisticated mathematics.

In nonrelativistic classical physics, the concept of energy emerges when one considers the power done (the scalar product of the force by the velocity) on a mass point or a volume element. First, in the schematic case of a mass point in a time-independent potential force field $V$, the energy of the mass point appears from the power equation as a natural conserved scalar quantity: the $\operatorname{sum}(1 / 2) m v^{2}+V$. That quantity is still relevant if the potential depends on time, but it is not constant any more. This is well known. In the more realistic case of a continuous medium subjected to internal forces and to an external force field, the energy is a volume density and it still emerges from the power done. However, in general, the local conservation of energy then appears in the form of a balance equation, though it is one in which there is no source term. That is, energy conservation means that the energy leaving or entering a given domain is exactly identified as a flux going through the boundary surface of the domain. This also is well known-see, for example, [1]. We illustrate the emergence of such a true conservation equation for a continuous medium in Section 2 by examining in detail the example of a self-gravitating system of deformable media with isentropic deformation in Newtonian gravity.

In relativistic theories (including relativistic quantum mechanics), on the other hand, the volume energy density is essentially the $\left(\begin{array}{ll}0 & 0\end{array}\right)$ component of the energy-momentumstress tensor, in what follows "the $T$-tensor" for brevity. The conservation-type equations verified by the $T$-tensor are discussed in nearly all textbooks about special or general relativity, of course. In Section 3, we recall why the conservation equation verified by the $T$-tensor in the Minkowski spacetime (see, e.g., Lifshitz and Landau [2] or Fock [3]) contains two true local conservation equations of the form found in the nonrelativistic example of Section 2 and why one may identify the density and flux in these two equations as those 
of energy and spatial momentum, respectively. We also note the dependence of the energy density and the energy flux on the reference frame. That dependence is a known fact (though a scarcely mentioned one), but often that fact is not well appreciated. Our new contribution here is to show that these quantities can nevertheless be given a rigorous meaning within a theory of general reference frames and the associated space manifolds.

As is well known, an expression of the T-tensor may be deduced when a Lagrangian is available, the latter being assumed to govern the relevant system of matter fields via the principle of stationary action (e.g., [2-9]). There are two distinct definitions of a T-tensor from a Lagrangian: (i) the so-called "canonical" or "Noether" tensor, say $\tau$, is a byproduct of the Euler-Lagrange equations and (ii) the "Hilbert tensor," say T, is the symmetric tensor obtained as the derivative of the Lagrangian density with respect to variations of the (space-time) metric. In Section 4, we review the definitions of the canonical and Hilbert tensors from a Lagrangian through the principle of stationary action in general space-time. We recall two important but seemingly not widely known cases where the "canonical tensor" is, or is not, a tensor. Then, we prove precise results regarding the definition of the Hilbert tensor field (Theorem 1). In doing so, we formulate sufficient conditions of regularity for the bounded set in which the action is calculated; we define exact boundary conditions to be verified by the infinitesimal coordinate change; and we give a detailed derivation of the equations. We do not need to use complex notions of fibre bundles. To our knowledge, such a relatively elementary but detailed proof is not available in the literature. Next, we recall the meaning of the standard conservation equation verified by the Hilbert tensor: we argue that one actually needs local definitions of the energy and momentum densities and their fluxes, in short a local definition of the T-tensor, and one needs also a local conservation equation for the energy. We briefly discuss a recent work that proposes a solution to the latter issue. We end Section 4 by stating and proving precise results regarding the uniqueness and the actual tensoriality of the Hilbert tensor (Theorem 2). In particular, we prove that the same variational equation applies when a complete variation of the sole metric is applied (65), as when the variation of the metric results from a mere coordinate change (or diffeomorphism) (54), although the meaning of these two equations is totally different; for example, the LHS of (54) is zero for an invariant Lagrangian. We prove that the variational equation (65) characterizes the components of the Hilbert tensor field-whence it follows that it is left unchanged by the addition of four-divergence. We also prove, in detail and by relatively elementary arguments, that this is indeed a (ll $\left.\begin{array}{ll}0 & 2\end{array}\right)$ tensor. This is just stated in the literature that we consulted, except for [7] which uses more advanced mathematics. Of course, it follows basically from the invariance of the action but, in our opinion, not in a fully trivial way.

Finally, in Section 5, we investigate whether the energy equation is unique for a given system of fields, that is, if the energy density and fluxes can be considered to be uniquely defined. We show that if the energy density and its flux depend on the fields (both the matter fields and the "long-distance" fields) in a polynomial way, then they are determined uniquely. We show this by considering separately the contributions of matter (including its potential energy in the long-distance fields) and the long-distance fields.

\section{Local Energy Conservation for an Isentropically Deformable Medium in Newtonian Gravity}

2.1. Local Energy Balance for the Matter Fields. Let us consider a deformable continuous medium, of mass density field $\rho$, having a general motion (including deformation and rotation), with velocity field $\mathbf{v}$, with respect to some inertial frame F; that is, $\mathbf{v}:=d \mathbf{x} / d t$, where $\mathbf{x}(X):=\left(x^{i}\right)_{i=1,2,3}$ is the spatial position associated with an event $X$ in the frame $\mathrm{F}$ and where $t \mapsto X(t)$ is the world line of a given "particle" of the medium, parameterized by the Newtonian time $t$. The internal force field in that medium is assumed to be described by the Cauchy stress tensor field $\boldsymbol{\sigma}$. We assume that this motion takes place in a gravitational field, with Newtonian gravity potential $U$. Newton's second law for a volume element of the medium is written as

$$
\rho \frac{d \mathbf{v}}{d t}=\rho \nabla U+\operatorname{div} \boldsymbol{\sigma}
$$

where $d / d t$ means the "material" (or "total") derivative: for a vector, ${ }^{1}$

$$
\frac{d \mathbf{v}}{d t}=\frac{\partial \mathbf{v}}{\partial t}+(\operatorname{grad} \mathbf{v})(\mathbf{v})
$$

The power (per unit volume) is obtained by taking the scalar product of (1) with the velocity v. On the new LHS, we have $\mathbf{h}(\mathbf{v}, d \mathbf{v} / d t)=(d / d t)\left(\mathbf{v}^{2} / 2\right)$, while, on the RHS, we note that

$$
\mathbf{v} \cdot \operatorname{div} \boldsymbol{\sigma}:=\mathbf{h}(\mathbf{v}, \operatorname{div} \boldsymbol{\sigma})=\operatorname{div} \boldsymbol{\sigma}(\mathbf{v})-\boldsymbol{\sigma}: \mathbf{D},
$$

where

$$
\mathbf{D}:=\frac{1}{2}\left(\operatorname{grad} \mathbf{v}+(\operatorname{grad} \mathbf{v})^{T}\right)
$$

is the strain rate tensor. ${ }^{2}$ We note also that $\mathbf{v} \cdot \nabla U=$ $d U / d t-\partial U / \partial t$. Still, we assume that the deformation of the continuum is isentropic, which means that the power of the internal forces is stored as the rate of elastic energy:

$$
\boldsymbol{\sigma}: \mathbf{D}=\rho \frac{d \Pi}{d t},
$$

with $\Pi$ being the mass density of internal (elastic) energy in the continuous medium. This assumption applies, in particular, to any elastic medium and also [3] to any barotropic perfect fluid, as is commonly assumed in astrophysics-a barotropic fluid is not plainly an elastic medium in the sense that it does not have a reference configuration. We thus get

$$
\rho \frac{d}{d t}\left(\frac{\mathbf{v}^{2}}{2}\right)=\rho\left(\frac{d U}{d t}-\frac{\partial U}{\partial t}\right)+\operatorname{div} \boldsymbol{\sigma}(\mathbf{v})-\rho \frac{d \Pi}{d t} .
$$


It suggests itself to put together the terms containing an exact total derivative:

$$
\rho \frac{d}{d t}\left(\frac{\mathbf{v}^{2}}{2}+\Pi-U\right)=-\rho \frac{\partial U}{\partial t}+\operatorname{div} \boldsymbol{\sigma}(\mathbf{v}) .
$$

On the RHS, we have a source term due to the external force field, plus a flux term. On the LHS, we have

$$
e_{m}:=\frac{\mathbf{v}^{2}}{2}+\Pi-U
$$

Using the continuity equation that expresses the mass conservation

$$
\frac{\partial \rho}{\partial t}+\operatorname{div}(\rho \mathbf{v})=0
$$

we easily get the well known fact that (for whatever scalar function $e_{m}$, actually)

$$
\begin{aligned}
\rho \frac{d e_{m}}{d t} & :=\rho\left(\frac{\partial e_{m}}{\partial t}+\mathbf{v} \cdot \nabla e_{m}\right) \\
& =\frac{\partial\left(\rho e_{m}\right)}{\partial t}+\operatorname{div}\left(\rho e_{m} \mathbf{v}\right) .
\end{aligned}
$$

From (8) and (10), we may rewrite (7) as

$$
\frac{\partial w_{m}}{\partial t}+\operatorname{div} \Phi_{m}=-\rho \frac{\partial U}{\partial t}
$$

with

$$
\begin{aligned}
& w_{m}:=\rho e_{m}=\rho\left(\frac{\mathbf{v}^{2}}{2}+\Pi-U\right), \\
& \boldsymbol{\Phi}_{m}:=w_{m} \mathbf{v}-\boldsymbol{\sigma}(v) .
\end{aligned}
$$

That is, we got a balance equation with an external source term on the RHS. The scalar field $w_{m}$ is thus the volume energy density of matter, including its potential energy in the gravitational field, and the spatial vector field $\Phi_{m}$ is thus (the surface density of) the matter energy flux. Equation (11) can be found in the literature; see Eq. (66.11) in Fock [3]. But its detailed derivation illustrates well the emergence of a balance equation for a continuous medium.

2.2. Balance for the Gravitational Field and Local Energy Conservation Equation. Now, we assume that all of the matter that produces the gravitational field is indeed in the form of isentropically deformable continuous media. (Of course, the characteristics of the media may vary in space.) Thus, the point-dependent mass density $\rho$ is just the source of the gravitational field. It therefore obeys the gravitational field equation, that is, the Poisson equation:

$$
\Delta U=-4 \pi G \rho .
$$

By using Cartesian coordinates, for which we have $\Delta U=U_{, i, i}$, one checks easily that (13) implies the following:

$$
\frac{\partial w_{g}}{\partial t}+\operatorname{div} \Phi_{g}=\rho \frac{\partial U}{\partial t}
$$

where

$$
w_{g}:=\frac{(\nabla U)^{2}}{8 \pi G}
$$

is the volume energy density of the gravitational field and

$$
\Phi_{g}:=-\frac{\partial U}{\partial t} \frac{\nabla U}{4 \pi G}
$$

is the gravitational energy flux. Equation (14) may be termed the energy balance equation of the gravitational field. Like (11), this also is a balance equation with a source term. The source term in (14) is just the opposite of the source term in (11). Therefore, combining (11) with (14), we get the local energy conservation equation in Newtonian gravity [10]:

$$
\frac{\partial w}{\partial t}+\operatorname{div} \boldsymbol{\Phi}=0
$$

with the total energy density $w:=w_{m}+w_{g}$ and the total energy flux $\Phi:=\Phi_{m}+\Phi_{g}$. Equation (17) is the standard form for true local conservation of energy in a continuum, with the definition of the field variables $w$ and $\Phi$ depending on the particular theory. It has essentially the same form as the continuity equation (9). There is also a local conservation equation for momentum in Newtonian gravity, and global (integral) conservation laws can also be derived; see, for example, [10, 11]. Strangely enough, however, we did not see in the literature the local equation (17) for an elastic medium or a barotropic fluid in Newtonian gravity (thus with definitions (12) and (15)-(16)). For instance, it is not there in the references quoted in the present paper. (The $i=0$ component of Eq. (13) in [11] is just the continuity equation (9), with $\rho$ being indeed the (Newtonian) density of mass; thus, it is not the conservation equation for the Newtonian energy, but the one for the mass.)

\section{Local Conservation Equations and the Energy-Momentum Tensor in Minkowski Space-Time}

Recall that the energy-momentum tensor of a continuum or a system of fields is a second-order space-time tensor field $\mathbf{T}$, preferably symmetric. In the Minkowski space-time, $\mathbf{T}$ verifies [2] the local conservation equation

$$
T_{, \nu}^{\mu \nu}=0 \quad \text { (in Cartesian coordinates). }
$$

(Here, Cartesian coordinates are now ones such that the space-time metric has components $g_{\mu \nu}=\eta_{\mu \nu}$, where the matrix $\left(\eta_{\mu \nu}\right):=\operatorname{diag}(1,-1,-1,-1)$.) It is easy to see that (18) is the conjunction of two conservation equations having the standard form (17). One is precisely the scalar conservation equation (17):

$$
\frac{\partial w}{\partial t}+\operatorname{div} \boldsymbol{\Phi}=0 \quad\left(t:=\frac{x^{0}}{c}\right)
$$


in which now (the tensor $\mathbf{T}$ being taken in mass units as in Fock [3])

$$
\begin{aligned}
& w:=c^{2} T^{00} \\
& \left.\Phi:=c^{3} T^{0 i} \partial_{i} \quad \text { (sum over } i=1,2,3\right) .
\end{aligned}
$$

The other conservation equation involved in (18) and having the form of (17) is a (spatial) vector equation:

$$
\frac{\partial \mathbf{P}}{\partial t}+\operatorname{div} \mathbf{\Sigma}=0
$$

where

$$
\begin{aligned}
& \mathbf{P}:=c T^{i 0} \partial_{i}, \\
& \Sigma:=c^{2} T^{i j} \partial_{i} \otimes \partial_{j} .
\end{aligned}
$$

We may integrate either of the two conservation equations (19) and (21) in any bounded spatial domain $\Omega$ (the integrability in an unbounded domain being not guaranteed). This gives us two integral conservation equations:

$$
\begin{aligned}
& \frac{d}{d t}\left(\int_{\Omega} w d V\right)=-\int_{\partial \Omega} \boldsymbol{\Phi} \cdot \mathbf{n} d S, \\
& \frac{d}{d t}\left(\int_{\Omega} \mathbf{P} d V\right)=-\int_{\partial \Omega} \boldsymbol{\Sigma} \cdot \mathbf{n} d S .
\end{aligned}
$$

Thus, in (23) and (24), the change on the LHS is due to the flux through the boundary $\partial \Omega$ on the RHS. The scalar $w$ is interpreted as the volume density of energy, and the spatial vector $\mathbf{P}$ is interpreted as the volume density of momentum [2]. Therefore, in view of (23) and (24), the spatial vector $\boldsymbol{\Phi}$ is interpreted as the surface density of the energy flux, and the spatial tensor $\Sigma$ is interpreted as the surface density of the momentum flux. This interpretation may be justified in several ways, notably the following two [3].

First, we examine the nonrelativistic limit for a barotropic perfect fluid or an elastic solid. In the second approximation, $w, \boldsymbol{\Phi}, \mathbf{P}$, and $\boldsymbol{\Sigma}$ in (20) and (22) have then the following expressions ([3, Sect. 32]):

$$
\begin{aligned}
w & :=c^{2} T^{00} \simeq c^{2} \rho+\frac{1}{2} \rho \mathbf{v}^{2}+\rho \Pi, \\
\boldsymbol{\Phi} & :=c^{3} T^{0 i} \partial_{i}=c^{3} T^{i 0} \partial_{i}=c^{2} \mathbf{P} \\
& \simeq c^{2} \rho \mathbf{v}+\left(\frac{1}{2} \rho \mathbf{v}^{2}+\rho \Pi\right) \mathbf{v}-\boldsymbol{\sigma}(\mathbf{v}), \\
\boldsymbol{\Sigma} & :=c^{2} T^{i j} \partial_{i} \otimes \partial_{j} \simeq \rho \mathbf{v} \otimes \mathbf{v}-\boldsymbol{\sigma} .
\end{aligned}
$$

Therefore, at the first approximation, the special-relativistic local energy conservation (19) reduces to the continuity equation (9), and at the second approximation it expresses the conservation of that rest-mass energy corrected by adding the conserved Newtonian energy ((17) with $U \equiv 0)$. Also, at the first approximation, the special-relativistic momentum conservation (21) reduces to the Newtonian momentum conservation equation in the absence of external field:

$$
\frac{\partial(\rho \mathbf{v})}{\partial t}+\operatorname{div}(\rho \mathbf{v} \otimes \mathbf{v}-\boldsymbol{\sigma})=0
$$

Second, we recognize in $w$ and $\mathbf{P}$, for the electromagnetic field, the usual definition of the electromagnetic energy density and the Poynting vector from the relevant expression of T ([3, Sect. 33]).

One does not use the symmetry of the tensor $\mathbf{T}$ to derive (19)-(21) and (23)-(24). If that symmetry is true, it implies that $\boldsymbol{\Phi}=c^{2} \mathbf{P}$ is true generally: the density of energy flux is equal to $c^{2}$ times the density of momentum. The same equations (23) and (24) apply also to Newtonian gravity as follows from (17) and the gravitational extension [10, 11] of (26).

As it is easy to check, under a purely spatial change of the chart (coordinate system),

$$
\begin{aligned}
& x^{\prime j}=f^{j}\left(\left(x^{k}\right)\right) \quad(j, k=1,2,3)\left(\text { or } \mathbf{x}^{\prime}=\mathbf{f}(\mathbf{x})\right), \\
& x^{\prime 0}=x^{0},
\end{aligned}
$$

the energy density $w$ (see $(20)_{1}$ ) is an invariant scalar, while $\boldsymbol{\Phi}$ (see $(20)_{2}$ ) and $\mathbf{P}$ (see $\left.(22)_{1}\right)$ transform indeed as spatial vectors, and $\boldsymbol{\Sigma}$ (see $\left.(22)_{2}\right)$ transforms indeed as a (2 0$)$ spatial tensor. One may give a rigorous geometric meaning to such "spatial" objects by defining a relevant space manifold $\mathrm{M}_{\mathrm{F}}$ as follows [12]. In general space-time, one can formally define a reference frame $\mathrm{F}$ as being an equivalence class of charts having the same domain of definition $U$ (an open subset of the space-time manifold V) and exchanging by a coordinate change ("transition map") having the form of (27). Let $P_{S}$ : $\mathbb{R}^{4} \rightarrow \mathbb{R}^{3}, \mathbf{X}:=\left(x^{\mu}\right) \mapsto \mathbf{x}:=\left(x^{j}\right)$ be the "spatial projection." The elements (points) of the space manifold $\mathrm{M}_{\mathrm{F}}$ are the world lines, each of which is the set of events that have a given spatial projection $\mathbf{x}$ in some chart $\chi: U \rightarrow \mathbb{R}^{4}, X \mapsto \mathbf{X}$, belonging to the class $\mathrm{F}$. That is, a world line $l$ is an element of $\mathrm{M}_{\mathrm{F}}$, iff there is a chart $\chi \in \mathrm{F}$ and a triplet $\mathbf{x} \in P_{S}(\chi(\mathrm{U}))$, such that $l$ is the set of all events $X$ in the domain $U$, whose spatial coordinates are $\mathbf{x}$ :

$$
l:=\left\{X \in \mathrm{U} ; P_{S}(\chi(X))=\mathbf{x}\right\} .
$$

It results easily from (27) that (28) holds true then in any chart $\chi^{\prime} \in \mathrm{F}$, of course with the transformed spatial projection triplet $\mathbf{x}^{\prime}=\mathbf{f}(\mathbf{x}):=\left(f^{j}(\mathbf{x})\right)$ [12]. For any chart $\chi \in \mathrm{F}$, one defines the "associated chart" as the mapping which associates, with a world line $l \in \mathrm{M}_{\mathrm{F}}$, the constant triplet of the spatial coordinates of the events $X \in l$ :

$$
\begin{aligned}
\tilde{\chi}: \mathrm{M} & \longrightarrow \mathbb{R}^{3}, \\
l & \longmapsto \mathbf{x} \quad \text { such that } \forall X \in l, \\
P_{S}(\chi(X)) & =\mathbf{x} .
\end{aligned}
$$

The set $\mathrm{M}_{\mathrm{F}}$ is endowed with a natural structure of threedimensional differentiable manifold, of which the basic atlas is made of the associated charts $\tilde{\chi}$, where $\chi$ is any chart belonging to the reference frame F [12]. The "spatial" objects are defined above: the scalar $w$, the vectors $\boldsymbol{\Phi}, \mathbf{P}$, and the tensor $\Sigma$ are simply and rigorously tensor fields on the manifold $\mathrm{M}_{\mathrm{F}}$. (Of course, they have in general, in addition, dependence on the time coordinate $x^{0}$; thus they are, strictly 
speaking, one-parameter families of tensor fields on $\mathrm{M}_{\mathrm{F}}$.) Fixing a reference frame in this sense can be done, for instance, by choosing one local coordinate system (chart $\chi$ ) on the space-time, with its domain of definition $\mathrm{U}$ : the corresponding reference frame $\mathrm{F}$ is then the equivalence class of this chart. As soon as one has fixed a reference frame, then (19) and (21), as well as (23) and (24), are coordinatefree equations on the space manifold $\mathrm{M}_{\mathrm{F}}$. In particular, the bounded spatial domain $\Omega$ is an open subset of the manifold $\mathrm{M}_{\mathrm{F}}$, having a regular boundary $\partial \Omega$, so that the divergence theorem applies. (See the Appendix for a precise definition of the needed regularity.)

On the other hand, if one makes a general coordinate change for which the change in the spatial coordinates depends on the time coordinate (already if one makes a Lorentz transformation transforming the Cartesian system into another one, but with a nonzero "boost"), then (20) defines completely different quantities $w^{\prime}$ and $\boldsymbol{\Phi}^{\prime}$, as compared with the initial ones. The same is true for $\mathbf{P}$ and $\boldsymbol{\Sigma}$ as defined by (22). This means that there is one definition of the energy and momentum (and their fluxes) per reference frame. It is not specific to special relativity. Indeed, the energy depends on the reference frame. This is true in nonrelativistic physics (e.g., [10]) - as may be checked here on the fact that $w_{m}$ and $\boldsymbol{\Phi}_{m}$ defined in (12) involve the velocity $\mathbf{v}$ that depends on the inertial frame, whereas $\rho, \Pi, U$, and $\sigma$ are Galilean invariants. It is also true in relativistic physics and also in general space-time, be it for the classical or the quantummechanical energy [13].

\section{Definition of the Energy-Momentum Tensor from a Lagrangian}

4.1. Lagrangian and Stationary Action Principle. We assume that the equations of motion for some "matter fields" $\phi^{A}(A=$ $1, \ldots, n)$ derive from a Lagrangian $L$ through the principle of stationary action in general space-time:

$$
\begin{aligned}
& \text { For any variation field } \delta \phi^{A}=\delta \phi^{A}(X) \\
& \qquad \begin{aligned}
\text { with } \delta \phi_{\mid \partial \mathrm{U}}^{A} & =0, \\
\text { we have } \delta S & =0 .
\end{aligned}
\end{aligned}
$$

Here, $\partial \mathrm{U}$ is the boundary, assumed smooth, of some bounded open set $U$ in the space-time, and $S$ is the action: in some chart $\chi$ whose domain of definition $\mathrm{W}$ contains $\mathrm{U}$, it is written as

$$
S=S_{\mathrm{U}}:=\int_{\chi(\mathrm{U})} L\left(\phi^{A}(\mathbf{X}), \phi_{, \mu}^{A}(\mathbf{X}), \mathbf{X}\right) \sqrt{-g(\mathbf{X})} d^{4} \mathbf{X},
$$

where $\phi^{A}: \mathbf{X} \mapsto \phi^{A}(\mathbf{X}), \chi(\mathrm{W}) \rightarrow \mathbb{R}^{n_{A}}$, is the local expression of the field $\phi^{A}$ in the chart $\chi$, and $g:=\operatorname{det}\left(g_{\mu \nu}\right)$, with $g_{\mu \nu}$ 's being the components of the metric tensor in the chart $\chi$; note that $\chi(\mathrm{W})$ is an open subset of $\mathbb{R}^{4}$. Thus, the field $\phi^{A}$ has $n_{A}$ real components (or $n_{A}$ complex components for a complex field, with $\mathbb{R}^{n_{A}}$ replaced by $\mathbb{C}^{n_{A}}$ ). At this stage, we do not need to know the exact geometric nature of the fields, whether they are scalars, vectors, more general tensors, or otherwise. We just assume that, on changing the chart: $\chi \hookrightarrow \chi^{\prime}$, the local expression of each of them has some definite transformation law, say $\phi^{A}(\mathbf{X}) \hookrightarrow \phi^{\prime A}\left(\mathbf{X}^{\prime}\right),{ }^{3}$ and that the Lagrangian is then invariant under the coordinate change:

$$
\begin{array}{r}
L\left(\phi^{\prime A}\left(\mathbf{X}^{\prime}\right), \phi_{, \mu}^{\prime A}\left(\mathbf{X}^{\prime}\right), \mathbf{X}^{\prime}\right) \\
\quad=L\left(\phi^{A}(\mathbf{X}), \phi_{, \mu}^{A}(\mathbf{X}), \mathbf{X}\right), \\
\mathbf{X}^{\prime}=\chi^{\prime}\left(\chi^{-1}(\mathbf{X})\right):=F(\mathbf{X}) .
\end{array}
$$

That invariance has to be true at least when the chart belongs to some well-defined class and implies that the same invariance is valid for action (31). In this section, we will consider the usual case that all charts (in the atlas of the space-time manifold) are allowed; that is, we will discuss generally covariant theories. However, it also makes sense to consider instead the class associated with a particular ("privileged") reference frame. Thus, the Lagrangian is a smooth real function $L=L\left(\mathbf{q}^{A}, \mathbf{q}_{\mu}^{A}, \mathbf{X}\right)$, where $\mathbf{X} \in \mathbb{R}^{4}$ is the coordinate vector specifying the space-time position, $\mathbf{q}^{A} \in \mathbb{R}^{n_{A}}$, and also $\mathbf{q}_{\mu}^{A} \in \mathbb{R}^{n_{A}}$ for $\mu=0, \ldots, 3$. These five vectors of $\mathbb{R}^{n_{A}}$ specify the values that may be taken at $\mathbf{X}$ by the local expression of the field $\phi^{A}$ and its partial derivatives. This means that, in expression (31) of the action, one makes the substitution

$$
\begin{aligned}
& \mathbf{q}^{A}=\phi^{A}(\mathbf{X}), \\
& \mathbf{q}_{\mu}^{A}=\phi_{, \mu}^{A}(\mathbf{X}) \\
& \quad(A=1, \ldots, n ; \mu=0, \ldots, 3) .
\end{aligned}
$$

Note also that $d V_{4}:=\sqrt{-g} d^{4} \mathbf{X}$, with $g:=\operatorname{det}\left(g_{\mu \nu}\right)$, is the invariant four-volume element on the space-time (thus, $g<0$ for a Lorentzian metric on the four-dimensional space-time).

Stationarity (30) is equivalent to the Euler-Lagrange equations (see, e.g., $[2,14]$; $\delta S$ is defined from a Gateaux derivative, as with (38)). In general space-time, the latter equations are written as [9]

$$
\partial_{\mu}\left(\frac{\partial \mathscr{L}}{\partial \mathbf{q}_{\mu}^{A}}\right)=\frac{\partial \mathscr{L}}{\partial \mathbf{q}^{A}} \quad(A=1, \ldots, n), \mathscr{L}:=L \sqrt{-g}
$$

in $\mathrm{U}$, with the implicit assignment (33). The domain of definition $\mathrm{W}$ of the coordinate system now has to contain not only $\mathrm{U}$ but also the boundary $\partial \mathrm{U}$, because the derivation of (34) needs to use the divergence theorem.

4.2. The "Canonical" (or "Noether") T-Tensor. We will give only a very brief account (see, e.g., [2, 4, 7-9]). This object has the following expression in a given chart:

$$
\begin{aligned}
\tau_{\mu}^{\nu}(\mathbf{X})= & \boldsymbol{\phi}_{, \mu}^{A}(\mathbf{X})\left(\frac{\partial L}{\partial \mathbf{q}_{\nu}^{A}}\right)_{\mathbf{q}^{B}=\phi^{B}(\mathbf{X}), \mathbf{q}_{\rho}^{B}=\phi_{\rho}^{B}(\mathbf{X})} \\
& -\delta_{\mu}^{\nu} L\left(\phi^{A}(\mathbf{X}), \boldsymbol{\phi}_{, \rho}^{A}(\mathbf{X}), \mathbf{X}\right) .
\end{aligned}
$$


When $-g=1$ and $\mathscr{L}=L=L\left(\mathbf{q}^{A}, \mathbf{q}_{\mu}^{A}\right)$ does not depend explicitly on the space-time position, this object occurs naturally from the derivation of the Euler-Lagrange equations (34), which imply that it verifies the desired local conservation equation $\tau_{\mu, \nu}^{\nu}=0$ [2]. However, such independence happens in practice only in flat space-time. Moreover, in fact, this object is not necessarily a tensor-even in flat space-time (cf. the case of the electromagnetic field [9]), with $\mathbf{F}$ being the field tensor and $\mathbf{A}$ the 4-potential, we have [2, 7]

$$
4 \pi \tau_{\mu}^{\nu}=-A_{\rho, \mu} F^{\nu \rho}+\frac{1}{4}\left(F_{\rho \sigma} F^{\rho \sigma}\right) \delta_{\mu}^{\nu} .
$$

(Henceforth, indices are raised or lowered with the spacetime metric.) On the RHS, everything, but $A_{\rho, \mu}$, is tensorial; hence, $\tau_{\mu}{ }^{\nu}$ is not a tensor; that is, $\tau_{\mu}{ }^{\nu}$ does not transform as a (1 1) tensor for general coordinate changes. Of course, this does not mean that there is no energy-momentum tensor for the electromagnetic field. (The Hilbert tensor indeed does the job; see, e.g., [2].) But it proves that the "canonical tensor" is not necessarily a tensor. This is not often noted; for example, it is not in [2,7], probably because (36) does behave as a (1 1 ) tensor for linear coordinate changes, as are the Lorentz transformations to which one often restricts oneself in special relativity. In general space-time, $\boldsymbol{\tau}$ is a tensor for a scalar field [9] and also for the Dirac field [15].

4.3. Hilbert's Variational Definition of the T-Tensor. While following the line of the classic derivation by Lifshitz and Landau [2], we will include many mathematical details which appear necessary in that derivation and that, for the most part, we did not find in the literature that we consulted. By this, we do not mean the geometric formulation of the physical fields as sections of appropriate fibre bundles, which has been implemented in [7], among others, and rather extensively in [8] - and which we will not need. (See the remarks following definition (31) of the action.) Instead, we mean the precise definition of "the variation of the action under an infinitesimal diffeomorphism," the regularity of the boundary and the exact boundary conditions, and a clear derivation of the main formulas.

One considers a given chart $\chi: X \mapsto \mathbf{X}=\left(x^{\mu}\right)$ and one imposes a small change to it: $x^{\mu} \hookrightarrow x^{\mu}+\delta x^{\mu}$. As we will see, the domain of definition $\mathrm{W}$ of $\chi$ must include the closure $\overline{\mathrm{U}}$ of the bounded open set $\mathrm{U}$ in which one computes action (31), and we must assume that $\delta x^{\mu}=0$ at the events $X \in \mathrm{W}$ which do not belong to $\mathrm{U}$. (Alternatively, one may regard the mapping defined in coordinates by $x^{\mu} \mapsto x^{\mu}+\delta x^{\mu}$ as a diffeomorphism of the space-time manifold $\mathrm{V}$, which coincides with the identity map for $X \notin \mathrm{U}$.) Thus, $\delta x^{\mu}=\epsilon \xi^{\mu}$ with $\xi$ any smooth vector field that vanishes if $X \notin U$ and $\epsilon \ll 1$. That is, we change the chart $\chi$ for a new chart $\chi_{\epsilon}$ given by

$$
\chi_{\epsilon}(X):=\mathbf{X}+\epsilon \boldsymbol{\xi}(\mathbf{X}) \quad X \in \mathrm{W}, \mathbf{X}:=\chi(X) .
$$

(The vector field $\mathbf{X} \mapsto \boldsymbol{\xi}(\mathbf{X})=\left(\xi^{\mu}(\mathbf{X})\right)$ is the local expression of $\xi$ in the chart $\chi$.) After such a coordinate change, the local expressions of the fields change, each according to its specific transformation behaviour, and the domain $\chi(\mathrm{U}) \subset \mathbb{R}^{4}$ also changes, so both the integrand and the integration domain change in action (31), which thus takes a priori a different value. One seeks to calculate the first-order term, as $\epsilon \rightarrow$ 0 , in the variation of the action, $S_{U}$ being considered as a functional of the field $\mathbf{X} \mapsto \delta \mathbf{X}=\epsilon \xi(\mathbf{X})$. This amounts to calculate the Gateaux derivative of $S_{\mathrm{U}}$ in the direction of the vector field $\xi$ :

$$
\frac{\left(\delta S_{U}\right)(\epsilon)}{\epsilon}:=\left(\frac{d}{d \epsilon} S_{U}(\epsilon \xi)\right)_{\epsilon=0} .
$$

The bounded open set $\mathrm{U}$ is assumed to be an open domain in the sense of Proposition A.3 in the Appendix; in particular, it has a smooth boundary $\partial \mathrm{U}$. Because $\mathrm{U}$ is an open domain, the assumption " $\xi(X)=0$ if $X \notin U$ " means exactly that the support of $\xi$ is included in the closure $\overline{\mathrm{U}}=\mathrm{U} \cup \partial \mathrm{U}$ (Corollary to Proposition A.3). ${ }^{4}$ Since $\operatorname{Supp}\left(\partial_{y} \xi^{\mu}\right) \subset \operatorname{Supp}\left(\xi^{\mu}\right)$, this implies that all derivatives of $\xi$ also vanish if $X \notin U$. It follows that the corresponding change in $g^{\mu \nu}$, as determined to the first order in $\epsilon$ (Eq. (94.2) in [2]),

$$
\delta g^{\mu \nu}=\epsilon\left(\xi^{\mu ; \nu}+\xi^{\nu ; \mu}\right)=\delta x^{\mu ; \nu}+\delta x^{\nu ; \mu},
$$

also vanishes if $X \notin \mathrm{U}$. (Here, $g^{\mu \nu}$ 's are the components of the inverse of the metric's component matrix $\left(g_{\mu \nu}\right)$.) Thus, in particular, $\delta g^{\mu \nu}=0$ on $\partial \mathrm{U}^{5}$

One assumes moreover that the matter Lagrangian $L$ depends on the matter fields and the metric and their firstorder derivatives, but not on their higher-order derivatives. The same applies then to the "Lagrangian density" $\mathscr{L}:=L \sqrt{-g}$; that is, the latter is a smooth real function $\mathscr{L}=\mathscr{L}\left(\mathbf{q}^{A}, \mathbf{q}_{\mu}^{A}, \underline{g}^{\mu \nu}, \underline{g}_{\rho}^{\mu \nu}\right)$. To calculate action (31), in the Lagrangian $L$, values (33) are assigned to $\mathbf{q}^{A}$ and $\mathbf{q}_{\mu}^{A}$. In addition, now one assigns the values $g^{\mu \nu}(\mathbf{X})$ and $g^{\mu \nu}{ }_{, \rho}(\mathbf{X})$ to $\underline{g}^{\mu v}$ and $\underline{g}_{\rho}^{\mu \nu}$, respectively. At this stage, it is usually admitted that the change in action $(31), \delta S_{U}$, is given by

$$
\begin{aligned}
\delta S_{\mathrm{U}} & =\int_{\chi(\mathrm{U})}\left[\frac{\partial \mathscr{L}}{\partial \mathbf{q}^{A}} \delta \phi^{A}+\frac{\partial \mathscr{L}}{\partial\left(\mathbf{q}_{\mu}^{A}\right)} \delta\left(\phi_{, \mu}^{A}\right)+\frac{\partial \mathscr{L}}{\partial \underline{g}^{\mu \nu}} \delta g^{\mu \nu}\right. \\
+ & \left.\frac{\partial \mathscr{L}}{\partial\left(\underline{g}_{\rho}^{\mu \nu}\right)} \delta\left(g_{, \rho}^{\mu \nu}\right)\right] d^{4} \mathbf{X},
\end{aligned}
$$

without a justification nor a precise definition of $\delta S_{U}, \delta \phi^{A}$, and so forth. (Actually, step (40) and some later intermediate steps are even skipped by Lifshitz and Landau [2].) However, recall that here not only the integrand but also the integration domain changes in action (31), so the first-order variation of the integral $S_{U}$ involves a priori more than just the firstorder approximation of the integrand. Let us be more precise. Denote provisionally the list of all fields (matter fields and metric) by $\phi^{J}(J=1, \ldots, n+1)$. Applying definition (31) with the new chart (37), we write

$$
S_{\epsilon}:=S_{U}(\epsilon \xi)=\int_{F_{\epsilon}(\Omega)} \widetilde{\mathscr{L}}(\mathbf{X}, \epsilon) d^{4} \mathbf{X},
$$


with $\Omega:=\chi(\mathrm{U})$,

$$
\begin{aligned}
F_{\epsilon} & :=\chi_{\epsilon} \circ \chi^{-1}, \\
\widetilde{\mathscr{L}}(\mathbf{X}, \epsilon) & :=\mathscr{L}\left(\phi_{\epsilon}^{J}(\mathbf{X}), \partial_{\mu} \phi_{\epsilon}^{J}(\mathbf{X})\right),
\end{aligned}
$$

where $\phi_{\epsilon}^{J}: \chi_{\epsilon}(\mathrm{W}) \rightarrow \mathbb{R}^{n_{J}}$ is the local expression of the field $\phi^{J}$ in the chart $\chi_{\epsilon}$. The integral (41) has a form that is well known, in particular, in continuum mechanics. The expression of its derivative is also well known:

$$
\frac{d S_{\epsilon}}{d \epsilon}=\int_{F_{\epsilon}(\Omega)}\left[\frac{\partial \widetilde{\mathscr{L}}}{\partial \epsilon}+\operatorname{div}\left(\widetilde{\mathscr{L}} \boldsymbol{\xi}_{\epsilon}\right)\right] d^{4} \mathbf{X},
$$

where $\boldsymbol{\xi}_{\epsilon}(\mathbf{X}):=\left(\partial F_{\epsilon} / \partial \epsilon\right)\left(\mathbf{X}_{0}\right)$, with $\mathbf{X}_{0}:=F_{\epsilon}^{-1}(\mathbf{X})$, is the "velocity field" at "time" $\epsilon$. In particular, we get from (37) and these definitions that $\boldsymbol{\xi}_{\epsilon=0}=\boldsymbol{\xi}$. Therefore, applying the divergence theorem in (43) and since $\xi=0$ on $\partial \mathrm{U}$ (hence $\boldsymbol{\xi}=\mathbf{0}$ on $\partial \Omega$, because $\partial \Omega=\partial(\chi(\mathrm{U}))=\chi(\partial \mathrm{U}))$, we get

$$
\begin{aligned}
& \left(\frac{d S_{\epsilon}}{d \epsilon}\right)_{\epsilon=0}=\int_{\Omega}\left(\frac{\partial \widetilde{\mathscr{L}}}{\partial \epsilon}\right)_{\epsilon=0} d^{4} \mathbf{X} \\
& =\int_{\Omega}\left[\frac{\partial \mathscr{L}}{\partial \mathbf{q}^{J}}\left(\frac{\partial\left(\phi_{\epsilon}^{J}(\mathbf{X})\right)}{\partial \epsilon}\right)_{\epsilon=0}\right. \\
& \left.+\frac{\partial \mathscr{L}}{\partial \mathbf{q}_{\mu}^{J}}\left(\frac{\partial\left(\partial_{\mu} \phi_{\epsilon}^{J}(\mathbf{X})\right)}{\partial \epsilon}\right)_{\epsilon=0}\right] d^{4} \mathbf{X} .
\end{aligned}
$$

Hence, defining simply the variations of the fields to the first order in $\epsilon$,

$$
\begin{aligned}
\delta \phi^{J}(\mathbf{X}) & :=\epsilon\left(\frac{\partial\left(\phi_{\epsilon}^{J}(\mathbf{X})\right)}{\partial \epsilon}\right)_{\epsilon=0}, \\
\delta\left(\phi_{, \mu}^{J}\right)(\mathbf{X}): & =\epsilon\left(\frac{\partial\left(\partial_{\mu} \boldsymbol{\phi}_{\epsilon}^{J}(\mathbf{X})\right)}{\partial \epsilon}\right)_{\epsilon=0},
\end{aligned}
$$

and remembering that $\phi^{\mathrm{J}}$ 's are the matter fields $\phi^{A}$ for $J=$ $A=1, \ldots, n$, while $\phi^{n+1}$ is the metric field $g^{\mu \nu}$, we see that (44), together with definition (38), proves the "obvious" (40). We see then from (43) that (40) is in general false if the boundary condition $\boldsymbol{\xi}=\mathbf{0}$ on $\partial \Omega$ is not valid.

If we write

$$
\left(\frac{\partial\left(\phi_{\epsilon}^{J}(\mathbf{X})\right)}{\partial \epsilon}\right)_{\epsilon=0}=\lim _{\epsilon \rightarrow 0} \frac{\phi_{\epsilon}^{J}(\mathbf{X})-\phi_{0}^{J}(\mathbf{X})}{\epsilon}
$$

and if we remember that $\phi_{\epsilon}^{J}$ is the local expression of the field $\phi^{J}$ in chart (37), which follows the flow of the vector field $\xi$ at small values of $\epsilon$, we recognize (at least in the case that $\phi^{J}$ is a tensor field) the definition of the Lie derivative [16] or rather of its opposite. That is,

$$
\delta \phi^{J}(\mathbf{X})=-\epsilon L_{\xi} \phi^{J}(\mathbf{X}) .
$$

We note also that

$$
\frac{\partial\left(\partial_{\mu} \phi_{\epsilon}^{J}(\mathbf{X})\right)}{\partial \epsilon}=\partial_{\mu}\left(\frac{\partial\left(\phi_{\epsilon}^{J}(\mathbf{X})\right)}{\partial \epsilon}\right) ;
$$

hence,

$$
\delta\left(\phi_{, \mu}^{J}\right)(\mathbf{X})=\partial_{\mu}\left(\delta \phi^{J}(\mathbf{X})\right) .
$$

In practical terms, $\delta \phi^{J}(\mathbf{X})$ can be computed in two steps [2]: first, one computes the difference $\phi_{\epsilon}^{J}\left(\mathbf{X}^{\prime}\right)-\phi^{J}(\mathbf{X})$ between the local expressions in the initial and the modified chart at points that correspond together through the transition map; that is, $\mathbf{X}^{\prime}=F_{\epsilon}(\mathbf{X})$. Second, one uses a first-order Taylor expansion to find the difference $\phi_{\epsilon}^{J}(\mathbf{X})-\phi^{J}(\mathbf{X})$, that is, at one and the same point. For instance, this gives indeed expression (39) for the metric [2]. As another example, consider a vector field $V$, with components $V^{\mu}$ in the initial chart. We find without difficulty

$$
\delta V^{\mu}=\delta x_{, \nu}^{\mu} V^{\nu}-V_{, \nu}^{\mu} \delta x^{\nu}=\delta x_{; \nu}^{\mu} V^{\nu}-V_{; \nu}^{\mu} \delta x^{\nu},
$$

where the second equality occurs due to the symmetry of the Christoffel symbols (i.e., due to the fact that the Levi-Civita connection has no torsion). This does coincide with the Lie derivative definition (47).

In a last step, let us assume that the matter fields obey the Euler-Lagrange equations (34). Just like for the derivation of the latter equations from the stationarity of action (31), because $\delta\left(\phi_{, \mu}^{A}\right)=\left(\delta \phi^{A}\right)_{, \mu}$ in view of (49), one may transform the second term in (40) and use the divergence theorem to make a surface term appear in it; and that surface term vanishes because $\delta \phi^{A}=0$ on $\partial \mathrm{U}$ (as can be checked for a vector on (50) and for the contravariant metric tensor on (39)). It then follows from (34) that the first two terms in (40) cancel one another; thus ${ }^{6}$

$$
\begin{array}{r}
\delta S_{\mathrm{U}}=\int_{\chi(\mathrm{U})}\left[\frac{\partial \mathscr{L}}{\partial \underline{g}^{\mu \nu}} \delta g^{\mu \nu}+\frac{\partial \mathscr{L}}{\partial\left(\underline{g}_{\rho}^{\mu \nu}\right)} \delta\left(g_{, \rho}^{\mu \nu}\right)\right] d^{4} \mathbf{X}, \\
\mathscr{L}:=L \sqrt{-g} .
\end{array}
$$

In the same way, because $\delta\left(g^{\mu \nu}, \rho\right)=\left(\delta g^{\mu \nu}\right)_{, \rho}$, one may transform the second term in (51) and use the divergence theorem to make a surface term appear in it, and that surface term vanishes because $\delta g^{\mu \nu}=0$ on $\partial \mathrm{U}$. One thus gets [2]

$$
\delta S_{\mathrm{U}}=\int_{\chi(\mathrm{U})}\left[\frac{\partial \mathscr{L}}{\partial \underline{g}^{\mu \nu}}-\frac{\partial}{\partial x^{\rho}}\left(\frac{\partial \mathscr{L}}{\partial\left(\underline{g}_{\rho}^{\mu \nu}\right)}\right)\right] \delta g^{\mu \nu} d^{4} \mathbf{X} .
$$

We have thereby proved the following.

Theorem 1. Assume that the bounded subset $\mathrm{U}$ of the spacetime is an open domain in the sense of Proposition A.3. In the domain $\mathrm{W} \supset \overline{\mathrm{U}}$ of some chart $\chi$, define a one-parameter family of new charts by (37), associated with a smooth vector 
field $\xi$ that is defined over $\mathrm{W}$ and vanishes for $X \notin \mathrm{U}$. Assume that the smooth matter Lagrangian has the form $L=$ $L\left(\mathbf{q}^{A}, \mathbf{q}_{\mu}^{A}, \underline{g}^{\mu \nu}, \underline{g}_{\rho}^{\mu \nu}\right)$. Then,

(i) the first-order variation $\delta S_{U}$ of the action, defined in (38), is given by (40). In this equation, the variations $\delta \phi^{J}$ and $\delta\left(\phi_{, \mu}^{J}\right)\left(J=1, \ldots, n+1\right.$, with $\left.\phi^{n+1}=\left(g^{\mu \nu}\right)\right)$ are defined by (45) or equivalently by (47) and (49);

(ii) if the matter fields $\phi^{A}(A=1, \ldots, n)$ obey the EulerLagrange equations (34), then $\delta S_{U}$ is given by (52) right above.

Equation (52) leads one to define an object $\mathbf{T}$ (usually called "Hilbert energy-momentum tensor," though not in [2]) by its components [2]:

$$
\begin{aligned}
& \frac{1}{2} \sqrt{-g} T_{\mu \nu}:=\frac{\partial \mathscr{L}}{\partial \underline{g}^{\mu \nu}}-\frac{\partial}{\partial x^{\rho}}\left(\frac{\partial \mathscr{L}}{\partial\left(\underline{g}_{\rho}^{\mu \nu}\right)}\right), \\
& \mathscr{L}:=L \sqrt{-g} .
\end{aligned}
$$

The symmetry of this object, $T_{\nu \mu}=T_{\mu \nu}$, follows from the symmetry of the metric and the invariance of $L$ under general coordinate changes. ${ }^{7}$ As shown by Theorem 1, for the object T whose components are defined by (53), we have "on shell" for any regular bounded open set $\mathrm{U}$ and for any coordinate change $\delta x^{\mu}=\epsilon \xi^{\mu}$ such that $\xi^{\mu}(X)$ vanishes for $X \notin \mathrm{U}$ :

$$
\delta S_{\mathrm{U}}=\frac{1}{2} \int_{\chi(\mathrm{U})} T_{\mu \nu} \delta g^{\mu \nu} \sqrt{-g} d^{4} \mathbf{X} .
$$

If the Lagrangian function $L$ is invariant under general coordinate changes, then the action $S_{U}$ in (31) is invariant too; hence, the change $\delta S_{U}$ given by (52) or (54) is zero for any possible coordinate change. Assume, moreover, that the object $\mathbf{T}$ given by (53) turns out to be indeed a tensor. (This tensorial character does not seem to be proved in the literature, but see Section 4.5.) Then, using expression (39) of $\delta g^{\mu \nu}$ in terms of the vector field $\xi$ and since by assumption the latter vanishes on $\partial \mathrm{U}$, one gets from (54) [2]

$$
T_{\mu ; \nu}^{v}=0
$$

4.4. Is (55) a True Conservation Equation? In contrast with (18) $\left(T_{\mu, v}^{v}=0\right.$, with partial derivatives), (55) (with covariant derivatives) "does not generally express any conservation law whatever" [2]. Fock [3] used similar words: he noted that the four scalar equations contained in (55) "do not by themselves lead to conservation laws." To explain it quickly, the presence of covariant derivatives gives to (55) the form of (18) plus source terms, which are the terms linear in the $T$-tensor itself (that involve the connection coefficients). Nevertheless, (55) can be rewritten in the form of (18) after introducing some "pseudotensor of the gravitational field" $\mathbf{t}$. But the definition of $\mathbf{t}$ is not unique. And $\mathbf{t}$ behaves as a tensor only for linear coordinate transformations. As a result, it is generally agreed that (55) can lead only (under special assumptions, e.g., asymptotically flat space-time) to global conservation laws; see, for example, [2, 17]. However, in order to be able to investigate the energy balance in any spatial domain, one would need to know uniquely the relevant energy density and its flux. And one would need that they obey a true and local conservation equation. (This is indeed the case in most fields of physics, e.g., in mechanics, thermodynamics, electrodynamics, and chemistry, as well as in Newtonian gravitation-as shown in Section 2-and also in several alternative relativistic theories of gravitation in flat space-time, e.g., [18-20], including a preferred-frame scalar theory with a mechanism for gravity [21].)

What is thus lacking in theories based on (55), which include general relativity and its numerous variants or extensions, is not merely an exact local concept of the gravitational energy. As we recalled, in special relativity, the local conservation equation (18) for the energy-momentum tensor can be rewritten as two local conservation equations of type (17): a scalar one for the energy and a vector one for the three-momentum. We believe that the local concept of energy is indissolubly bound with the existence of a true local conservation equation of type (17), as it exists both in nonrelativistic physics and in special relativity. Since, as we recalled, the rewriting of (55) as an exact local conservation equation having form (18) is neither tensorial nor unique, we consider that (55) does not provide an exact local concept for any form of material energy, either: assuming the definition of the Hilbert tensor $\mathbf{T}$ is unique (which is proved in Section 4.5), one could define the material energy density as, say, $w:=T^{00}$. But another one could prefer to choose $w^{\prime}=T_{00}$ and a third one $w^{\prime \prime}:=T_{0}^{0}$. In the absence of an exact local conservation equation of type (18), nobody can tell which choice is correct. Another point is of course that, for a given bounded spatial domain $\Omega$, the time evolution of, say, the integral of $w:=T^{00}$ (the LHS of (23)) is not equal to a flux through the boundary $\partial \Omega$ but is also affected by source terms depending on the gravitational field, for which there is no unique definition of the energy-momentum tensor.

However, according to Padmanabhan [22], there exists a suitable definition for what we will call an energy current fourvector $\mathscr{G}$ of the gravitational field, such that the total energy current $\mathscr{P}:=\mathscr{G}+\mathbf{P}$ is conserved:

$$
\mathscr{P}_{; \mu}^{\mu}=\mathscr{G}_{; \mu}^{\mu}+P_{; \mu}^{\mu}=0
$$

where the four-vector field $\mathbf{P}$, with components

$$
P^{\mu}:=+T^{\mu}{ }_{\nu} \nu^{\nu}
$$

is the matter energy current associated with the matter distribution, for the observer(s) having the four-velocity field v. (The plus sign is with the (+---) signature that we use. This four-vector $\mathbf{P}$ should not be confused with the three-vector $\mathbf{P}$ in $(22)_{1}$. Similarly, the four-vector $\mathbf{v}$ should not be confused with the three-vector $\mathbf{v}$ of Sections 2 and 3. The gravitational 
current $\mathscr{G}$ of [22] also depends on v.) Since, in coordinates adapted to the reference fluid defined by $\mathbf{v}$, we have $[12,23]$

$$
\begin{aligned}
& v^{0}=\frac{1}{\sqrt{g_{00}}}, \\
& v^{j}=0,
\end{aligned}
$$

we note that, in any such adapted coordinates,

$$
P^{\mu}=\frac{T_{0}^{\mu}}{\sqrt{g_{00}}} .
$$

In particular, in the Minkowski space-time and taking for $\mathbf{v}$ the four-velocity field of some inertial reference frame, the gravitational current $\mathscr{G}$ vanishes; hence, conservation (56) of the (matter) current is exactly the same equation as the local energy conservation (19) with definitions (20), whence our use of the expression "matter energy current" to designate the four-vector field (57). Thus, according to the result [22], the situation regarding the energy and momentum conservation would be nearly the same in GR as it is in the ether theory [21]; namely, (i) there is a (scalar) local conservation equation of type (17) for the total (material plus gravitational) energy; (ii) the energy density and its flux involved in that conservation equation depend on the reference frame; ${ }^{8}$ (iii) there is no equivalent local conservation equation for the spatial momentum (by this we mean a (frame-dependent) space vector equation, thus three scalar equations, as (21)). Anyway, as discussed in [21], the conservation of the total momentum, when it takes place, precludes conservation of the momentum of matter.

4.5. Uniqueness and Tensoriality of the Hilbert Tensor. In addition to the difficulty described in the foregoing subsection, which did not seem solvable in the framework of the said theories (but may be solved by the work [22]), there is a point that needs clarification. In curved space-time, the Hilbert tensor field $\mathbf{T}$ is taken as the source of the gravitational fieldin general relativity and in many other relativistic theories of gravity. Clearly, that source has to be locally defined: it is not the global value (the space integral) of $\mathbf{T}$ that matters to determine the gravitational field, but indeed the distribution of its local value. However, could not the Hilbert tensor be subject to "relocalizations" due to the fact that the Lagrangian determining the equations of motion is not unique?

Let us add to the Lagrangian $L$ a total divergence: $L \hookrightarrow$ $L^{\prime}=L+D$ with

$$
D=\operatorname{div} \mathbf{V}=\frac{1}{\sqrt{-g}} \partial_{\rho}\left(V^{\rho} \sqrt{-g}\right)
$$

where $V^{\mu}=V^{\mu}\left(\mathbf{q}^{A}, \mathbf{q}_{\mu}^{A}, \underline{g}^{\mu \nu}, \underline{g}_{\rho}^{\mu \nu}\right)$ is a space-time vector field. (Of course, the partial derivatives in (60) apply once the relevant fields have been substituted for the arguments of $V^{\mu}$; see before (40).) Then, the Euler-Lagrange equations (34) stay unchanged; see, for example, [14]. Note that, of course, the modified Lagrangian $L^{\prime}$ is also an invariant scalar if $L$ is. But, $a$ priori, should not the $T$-tensor generally change? This would indirectly contradict a statement of Forger and Römer [8], according to which the energy-momentum tensor field " $\mathbf{T}$ is the rank 2 tensor field on space-time $\mathrm{M}$ depending on the fields of the theory which satisfies

$$
\delta_{g} \int_{\mathrm{K}} d^{n} \mathbf{X} \sqrt{|\operatorname{det} g|} L=-\frac{1}{2} \int_{\mathrm{K}} d^{n} \mathbf{X} \sqrt{|\operatorname{det} g|} T^{\mu \nu} \delta g_{\mu \nu}
$$

for every compact subset K of M and for every variation $\delta g_{\mu \nu}$ of the metric tensor with support contained in K." (Here, $n$ is the dimension of the space-time; thus, $n=4$ as far as we know. For us, $\mathrm{K}$ is the compact closure of the bounded open domain $\mathrm{U}, \mathrm{K}:=\overline{\mathrm{U}}=\mathrm{U} \cup \partial \mathrm{U}$; see Note 4.) Indeed, from this statement, one easily concludes that a total divergence does not change $T^{\mu \nu}$ (see [8], and see a detailed proof here around (75)). However, we have the following:

(a) It is not precised what is meant exactly by a "variation $\delta g_{\mu \nu}$ of the metric tensor" and what is meant exactly by $\delta_{g}$ (applied to the action integral) in the statement reproduced above, included in Theorem 4.2 of [8]. In the arguments (pp. 360-361) which lead the authors to state that theorem, the same situation is considered as in Section 4.3. That is, the variation of the metric occurs due to an infinitesimal coordinate change (or equivalently due to an infinitesimal diffeomorphism), generated by a vector field $\xi$ (noted as $X$ in [8]), with support contained in $\mathrm{K}$. (The variation of the metric is thus appropriately noted as $\delta_{X} \mathcal{g}_{\mu \nu}$ in these arguments, and the variation of the action is noted as $\delta_{X} S_{K}$.) It is in this precise situation that one can at the same time derive the basic equation (52) and state that actually $\delta S_{\mathrm{U}}=0$-which is needed to derive the "covariant conservation" (55).

(b) In that situation (i.e., the variation of the metric resulting thus from a coordinate change), (61) is equivalent to (54). However, we do not see how it could be the case that the validity of (54) "for every (regular) compact subset $\mathrm{K}$ of $\mathrm{M}$ and for every variation $\delta g_{\mu \nu}$ of the metric tensor with support contained in $\mathrm{K}$ " would characterize (determine) some field object having components $T_{\mu \nu}$. Indeed, since the action is invariant under coordinate changes, it follows that the LHS of (54) is automatically zero (for every (regular) compact subset $\mathrm{K}$, etc.). Hence, for example, $T_{\mu \nu} \equiv 0$ is a solution of (54) (for every (regular) compact subset $\mathrm{K}$, etc.) as well as is (53).

(c) In that same situation, the following argument in [8] does not work. According to this argument, "T does not change when $L$ is modified by the addition of a total divergence $(\cdots)$, simply because the addition of such a term does not affect the LHS of (61)." The last statement is true, but since in that situation the LHS of (61) is always zero, it can not prove that $\mathbf{T}$ does not change.

(d) Therefore, it seems that, instead of the foregoing situation, the "variation $\delta g_{\mu \nu}$ of the metric tensor" 
alluded to in Theorem 4.2 of [8] is a variation of the metric itself (thus even in a fixed coordinate system):

$$
g_{(\epsilon \mathbf{h})}^{\mu \nu}(\mathbf{X})=g^{\mu \nu}(\mathbf{X})+\epsilon h^{\mu \nu}(\mathbf{X}),
$$

where the field $\mathbf{h}$, with components $h^{\mu \nu}$, is a given field of symmetric ( 200$)$ tensors defined on U. (The matter fields are thus left unchanged.) However, if that is indeed the case, then (61) has a different meaning than (54): for example, the variation $\delta g^{\mu \nu}$ of the metric now depends on the ten independent parameters $h^{\mu \nu}(0 \leq \mu \leq \nu \leq 3)$ instead of merely the four parameters $\xi^{\mu}(\mu=0, \ldots, 3)$ as is the case in the arguments which lead to the statement of Theorem 4.2 in [8], as well as in Section 4.3. Thus, the validity of (61) in that different situation has to be proved separately. This proof takes points (i) and (ii) of the following theorem, whose conclusive part is its point (iii).

Theorem 2. Let $L=L\left(\mathbf{q}^{A}, \mathbf{q}_{\mu}^{A}, \underline{g}^{\mu \nu}, \underline{g}_{\rho}^{\mu \nu}\right)$ be a Lagrangian that is defined and smooth whenever the determinant of the matrix $\left(g^{\mu \nu}\right)$ is negative and that is invariant under general coordinate changes. Let $\mathrm{U}$ be a bounded open domain of the space-time manifold $\mathrm{V}$ and let $\mathrm{K}=\overline{\mathrm{U}}=\mathrm{U} \cup \partial \mathrm{U}$ be its compact closure. Let $\mathrm{E}$ be the vector space of the symmetric (2 0$)$ tensor fields $\mathbf{g}$ which are defined and continuous on $\mathrm{K}$ and which are $\mathscr{C}^{1}$ on $\mathrm{U}$. Let $\mathrm{E}^{\star}$ be the subset of $\mathrm{E}$ made of the tensor fields $\mathbf{g} \in \mathrm{E}$ such that, for any chart $\chi$ defined in a neighborhood of $\mathrm{K}$ (assuming there do exist such charts), one has $g^{-1}:=\operatorname{det} G<0$ over the domain $\chi(\mathrm{K})$, where $G$ is the component matrix $G=\left(g^{\mu \nu}\right)$. The matter fields $\phi^{A}(A=1, \ldots, n)$, being given functions which are defined and continuous on $\mathrm{K}$ and which are $\mathscr{C}^{1}$ on $\mathrm{U}$, define an invariant functional S on $\mathrm{E}^{\star}$ by

$S(\mathbf{g})$

$$
\begin{array}{r}
:=\int_{\Omega} \mathscr{L}\left(\phi^{A}(\mathbf{X}), \phi_{, \mu}^{A}(\mathbf{X}), g^{\mu \nu}(\mathbf{X}), g_{, \rho}^{\mu \nu}(\mathbf{X})\right) d^{4} \mathbf{X}, \\
\Omega:=\chi(\mathrm{U}), \mathscr{L}:=\sqrt{-g} L .
\end{array}
$$

(i) Given any two tensor fields $\mathbf{g} \in \mathrm{E}^{\star}$ and $\mathbf{h} \in \mathrm{E}$, there is a number $a=a(\mathbf{g}, \mathbf{h})>0$ such that, for $\epsilon \in]-a,+a[$, the tensor field $\mathbf{g}+\epsilon \mathbf{h}$ is in $\mathrm{E}^{\star}$. One has

$$
\begin{aligned}
\Delta_{\mathbf{g h}} S & :=\left(\frac{d}{d \epsilon} S(\mathbf{g}+\epsilon \mathbf{h})\right)_{\epsilon=0} \\
& =\int_{\Omega}\left[\frac{\partial \mathscr{L}}{\partial \underline{g}^{\mu \nu}} h^{\mu \nu}+\frac{\partial \mathscr{L}}{\partial\left(\underline{g}_{\rho}^{\mu \nu}\right)} h_{, \rho}^{\mu \nu}\right]_{\mathbf{g}} d^{4} \mathbf{X},
\end{aligned}
$$

where the subscript $\mathbf{g}$ means that, at any $\mathbf{X} \in \Omega$, one considers the derivatives of the function $\mathscr{L}$ for the values $g^{\mu \nu}=$ $g^{\mu \nu}(\mathbf{X}), \underline{g}_{\rho}^{\mu \nu}=g_{, \rho}^{\mu \nu}(\mathbf{X})$ of its arguments.

(ii) If $\mathbf{g} \in \mathrm{E}^{\star}, \mathbf{h} \in \mathrm{E}$, and $\mathbf{h}(X)=0$ when $X \in \partial \mathrm{U}$, then one has, in any chart $\chi$ whose domain contains $\mathrm{K}$,

$$
\Delta_{\mathbf{g h}} S=\frac{1}{2} \int_{\Omega} T_{\mu \nu} h^{\mu \nu} \sqrt{-g} d^{4} \mathbf{X}
$$

where $T_{\mu \nu}$ 's are defined in (53), the derivatives being taken as in the subscript notation $\mathrm{g}$ above.

(iii) Equation (65) determines uniquely the continuous functions $\mathbf{X} \mapsto T_{\mu \nu}(\mathbf{X}), \chi(\mathrm{K}) \rightarrow \mathbb{R}$. It follows that the "Hilbert tensor" with components (53) is not modified by the addition of a four-divergence. Moreover, this is indeed a (0 2) tensor field.

Proof. As a preliminary, recall that if the matrix $G=\left(g^{\mu \nu}\right)$ of some $\left(\begin{array}{ll}2 & 0\end{array}\right)$ tensor field $\mathbf{g}$ in one chart $\chi$ verifies $g^{-1}:=$ $\operatorname{det} G<0$ over $\chi(\mathrm{K})$, then the corresponding matrix $G^{\prime}$ in any other chart $\chi^{\prime}$ in the atlas of $\mathrm{V}$, whose domain also contains $\mathrm{K}$, verifies $g^{\prime^{-1}}:=\operatorname{det} G^{\prime}<0$ over $\chi^{\prime}(\mathrm{K})$ : indeed, the Jacobian matrix $J=\left(\partial x^{\prime \mu} / \partial x^{\nu}\right)$ is invertible for these two compatible charts, and we have $g^{\prime-1}=g^{-1}(\operatorname{det} J)^{2}$. Also remember that the invariance of functional (63) under the change of the chart follows from the definition of a Lagrangian that is invariant under general coordinate changes (32) and from the invariance of the four-volume measure $d V_{4}:=\sqrt{-g} d^{4} \mathbf{X}$.

(i) Let $\mathbf{g} \in \mathrm{E}^{\star}$ and $\mathbf{h} \in \mathrm{E}$; thus, in particular, these are two continuous functions defined over the compact set $\mathrm{K}$. The real function $M \mapsto \phi(M):=\operatorname{det} M$ is defined and $\mathscr{C}^{1}$ is over the vector space $\mathrm{M}(4, \mathbb{R})$ of the real $4 \times 4$ matrices. Choose a chart $\chi$ defined in a neighborhood of $\mathrm{K}$. For $\mathbf{X} \in \chi(\mathrm{K})$, we note $G(\mathbf{X})$, the matrix $\left(g^{\mu \nu}(\mathbf{X})\right)$. Let us note also $H(\mathbf{X})=\left(h^{\mu \nu}(\mathbf{X})\right)$. Since $\mathbf{g} \in \mathrm{E}^{\star}$, we have $\phi(G(\mathbf{X}))<0$ for $\mathbf{X} \in \chi(\mathrm{K})$. Because $\phi \circ G$ is a continuous function over the compact $\chi(\mathrm{K})$, it is bounded and reaches its bounds. Hence, for some number $d<0$, we have $\phi(G(\mathbf{X})) \leq d$ for $\mathbf{X} \in \chi(\mathrm{K})$. The set of matrices $\mathrm{C}=\{M=$ $G(\mathbf{X})+\epsilon H(\mathbf{X}) ; \mathbf{X} \in \chi(\mathrm{K})$ and $|\epsilon| \leq 1\}$ is compact; hence, we have $\sup \left\{\left\|\phi^{\prime}(M)\right\| ; M \in \mathrm{C}\right\}=A<\infty$. Also, $\sup \{\|H(\mathbf{X})\| ; \mathbf{X} \in$ $\chi(\mathrm{K})\}=B<\infty$. Therefore, we have for any $\mathbf{X} \in \chi(\mathrm{K})$ and any $\epsilon \in]-1,1[$

$$
\begin{aligned}
& |\phi(G(\mathbf{X})+\epsilon H(\mathbf{X}))-\phi(G(\mathbf{X}))| \leq A|\epsilon|\|H(\mathbf{X})\| \\
& \quad \leq|\epsilon| A B .
\end{aligned}
$$

Hence, there is a number $a>0$ such that, for $\epsilon \epsilon]-a,+a$, we have $\phi(G(\mathbf{X})+\epsilon H(\mathbf{X}))<d / 2<0$ for any $\mathbf{X} \in \chi(\mathrm{K})$. Thus, for $\epsilon \in]-a,+a$ [, we have $\mathbf{g}+\epsilon \mathbf{h} \in \mathrm{E}^{\star}$, as announced. Since that statement does not depend on the chart, the number $a>0$ does not depend on the chart either; thus, $a=a(\mathbf{g}, \mathbf{h})>0$.

Denoting henceforth $\mathbf{g}_{(\epsilon \mathbf{h})}:=\mathbf{g}+\epsilon \mathbf{h}$ for brevity, we define a $\mathscr{C}^{1}$ function $f$ from $\Omega \times$ ] $-a,+a$ [ into $\mathbb{R}$ by setting

$$
\begin{aligned}
& f(\mathbf{X}, \epsilon) \\
& \quad=\mathscr{L}\left(\phi^{A}(\mathbf{X}), \phi_{, \mu}^{A}(\mathbf{X}), g_{(\epsilon \mathbf{h})}^{\mu \nu}(\mathbf{X}), \partial_{\rho} g_{(\epsilon \mathbf{h})}^{\mu \nu}(\mathbf{X})\right) .
\end{aligned}
$$

Using definition (62), we have

$$
\begin{aligned}
\frac{\partial\left(g_{(\epsilon \mathbf{h})}^{\mu \nu}(\mathbf{X})\right)}{\partial \epsilon} & =h^{\mu \nu}(\mathbf{X}) \\
\frac{\partial\left(\partial_{\rho} g_{(\epsilon \mathbf{h})}^{\mu \nu}(\mathbf{X})\right)}{\partial \epsilon} & =\frac{\partial}{\partial \epsilon}\left(g_{, \rho}^{\mu \nu}(\mathbf{X})+\epsilon h_{, \rho}^{\mu \nu}(\mathbf{X})\right) \\
& =h_{, \rho}^{\mu \nu}(\mathbf{X}) .
\end{aligned}
$$


Therefore, we get

$$
\begin{aligned}
\frac{\partial f}{\partial \epsilon}(\mathbf{X}, \epsilon)= & \left(\frac{\partial \mathscr{L}}{\partial \underline{g}^{\mu \nu}}\right)_{g_{(\epsilon \mathbf{h})}} \frac{\partial\left(g_{(\epsilon \mathbf{h})}^{\mu \nu}(\mathbf{X})\right)}{\partial \epsilon} \\
& +\left(\frac{\partial \mathscr{L}}{\partial\left(\underline{g}_{\rho}^{\mu \nu}\right)}\right)_{g_{(\epsilon \mathbf{h})}} \frac{\partial\left(\partial_{\rho} g_{(\epsilon \mathbf{h})}^{\mu \nu}(\mathbf{X})\right)}{\partial \epsilon} \\
= & \left(\frac{\partial \mathscr{L}}{\partial \underline{g}^{\mu \nu}}\right)_{g_{(\epsilon \mathbf{h})}} h^{\mu \nu}(\mathbf{X}) \\
& +\left(\frac{\partial \mathscr{L}}{\partial\left(\underline{g}_{\rho}^{\mu \nu}\right)}\right)_{g_{(\epsilon \mathbf{h})}} h_{, \rho}^{\mu \nu}(\mathbf{X}) .
\end{aligned}
$$

From definitions (63) and (67), we have $S\left(\mathbf{g}_{(\epsilon \mathbf{h})}\right)=\int_{\Omega} f(\mathbf{X}$, є) $d^{4} \mathbf{X}$; hence,

$$
\frac{d}{d \epsilon} S\left(\mathbf{g}_{(\epsilon \mathbf{h})}\right)=\int_{\Omega} \frac{\partial f}{\partial \epsilon}(\mathbf{X}, \epsilon) d^{4} \mathbf{X}
$$

Since at $\epsilon=0$ we have $\mathbf{g}_{(\epsilon \mathbf{h})}=\mathbf{g}$, (64) follows from (69) and (70).

(ii) The second term in the integrand on the RHS of (64) can be written as

$$
\begin{gathered}
\left(\frac{\partial \mathscr{L}}{\partial\left(\underline{g}_{\rho}^{\mu \nu}\right)}\right)_{\mathbf{g}} h_{, \rho}^{\mu \nu}(\mathbf{X}) \\
=\frac{\partial}{\partial x^{\rho}}\left(\left(\frac{\partial \mathscr{L}}{\partial\left(\underline{g}_{\rho}^{\mu \nu}\right)}\right)_{\mathbf{g}} h^{\mu \nu}(\mathbf{X})\right) \\
-h^{\mu \nu}(\mathbf{X}) \frac{\partial}{\partial x^{\rho}}\left(\frac{\partial \mathscr{L}}{\partial\left(\underline{g}_{\rho}^{\mu \nu}\right)}\right)_{\mathbf{g}}
\end{gathered}
$$

The first term on the RHS of (71) is a divergence in $\mathbb{R}^{4}$ and its integral on $\Omega$ vanishes if $h^{\mu \nu}(\mathbf{X})=0(\mu, \nu=0, \ldots, 3)$ on $\partial \Omega$, thus if $\mathbf{h}(X)=0$ on $\partial \mathrm{U}$. (Remember that $\partial \Omega=\partial(\chi(\mathrm{U}))=$ $\chi(\partial \mathrm{U})$.) Hence, in that case, (64) is rewritten as

$$
\Delta_{\mathbf{g h}} S=\int_{\Omega}\left[\frac{\partial \mathscr{L}}{\partial \underline{g}^{\mu \nu}}-\frac{\partial}{\partial x^{\rho}}\left(\frac{\partial \mathscr{L}}{\partial\left(\underline{g}_{\rho}^{\mu \nu}\right)}\right)\right]_{\mathbf{g}} h^{\mu \nu} d^{4} \mathbf{X} .
$$

In view of (53), this is (65).

(iii) Consider a given tensor field $\mathbf{g} \in \mathrm{E}^{\star}$ and, in a given chart $\chi$ whose domain contains $\mathrm{K}$, let $\mathbf{X} \mapsto T_{\mu \nu}(\mathbf{X})$ and $\mathbf{X} \mapsto$ $\widetilde{T}_{\mu \nu}(\mathbf{X})$ be two sets of functions $(\mu, \nu=0, \ldots, 3)$ defined and continuous over $\chi(\mathrm{K})$, each set being symmetric, such that both verify (65) for any tensor field $\mathbf{h} \in \mathrm{E}$ that vanishes on $\partial \mathrm{U}$.
(We do not assume that either $T_{\mu \nu}$ or $\widetilde{T}_{\mu \nu}$ is given by (53).) We claim that $T_{\mu \nu}=\widetilde{T}_{\mu \nu}$ over $\chi(\mathrm{K})$. Denoting $\delta T_{\mu \nu}:=\widetilde{T}_{\mu \nu}-T_{\mu \nu}$, we thus have for any such tensor field $\mathbf{h}$

$$
\int_{\Omega} \delta T_{\mu \nu} h^{\mu \nu} \sqrt{-g} d^{4} \mathbf{X}=0
$$

Consider a given pair $\left(\mu_{0}, \nu_{0}\right)$ of indices. Let $\varphi$ be any real function which is defined and continuous over $\chi(\mathrm{K})$, which is $\mathscr{C}^{1}$ over $\Omega$, and that has compact support $K^{\prime} \subset \Omega$. Hence, $\varphi(\mathbf{X}) \neq 0$ implies $\mathbf{X} \in \Omega$. If $\mathbf{X} \in \partial \Omega$, we have $\mathbf{X} \notin \Omega$ since $\Omega$ is open; hence, $\varphi(\mathbf{X})=0$. Therefore, by setting $h^{\mu \nu}=$ $(1 / 2)\left(\delta_{\mu_{0}}^{\mu} \delta_{v_{0}}^{\nu}+\delta_{v_{0}}^{\mu} \delta_{\mu_{0}}^{\nu}\right) \varphi$ in the chart $\chi$, we define a tensor field $\mathbf{h} \in \mathrm{E}$ such that $\mathbf{h}(X)=0$ for $X \in \partial \mathrm{U}$. We can thus apply (73) to get

$$
\begin{aligned}
\int_{\Omega} & \left(\delta T_{\mu_{0} \nu_{0}}+\delta T_{v_{0} \mu_{0}}\right) \sqrt{-g} \varphi d^{4} \mathbf{X} \\
= & \int_{\Omega}\left(2 \delta T_{\mu_{0} v_{0}} \sqrt{-g}\right) \varphi d^{4} \mathbf{X}=0 .
\end{aligned}
$$

(The second equality follows from the symmetry of $T_{\mu \nu}$ and $\widetilde{T}_{\mu \nu}$.) Since this is true for any such function $\varphi$, we deduce that $\delta T_{\mu_{0} \nu_{0}} \sqrt{-g}$ is zero almost everywhere in $\Omega$. But since this is a continuous function, it is zero everywhere in the open set $\Omega \subset$ $\mathbb{R}^{4}$, and therefore it is zero also in its closure $\overline{\chi(\mathrm{U})}=\chi(\overline{\mathrm{U}})=$ $\chi(\mathrm{K})$. Then, since $\sqrt{-g} \neq 0$ over $\chi(\mathrm{K})$, we have $\delta T_{\mu_{0} v_{0}}=0$ over $\chi(\mathrm{K})$. This proves our precise statement about uniqueness at the beginning of this paragraph.

Now suppose the Lagrangian is a four-divergence: $L=$ $\left(\left(\sqrt{-g} V^{\mu}\right)_{, \mu}\right) / \sqrt{-g}$ with $V^{\mu}=V^{\mu}\left(\mathbf{q}^{A}, \mathbf{q}_{\mu}^{A}, \underline{g}^{\mu \nu}, \underline{g}_{\rho}^{\mu \nu}\right)$. Then, integral (63) is rewritten as

$$
\begin{aligned}
& S(\mathbf{g}+\epsilon \mathbf{h})=\int_{\partial \Omega} U^{\mu}\left(\phi^{A}(\mathbf{X}), \phi_{, \mu}^{A}(\mathbf{X}), g_{(\epsilon \mathbf{h})}^{\mu \nu}(\mathbf{X})\right. \\
& \left.\left(g_{(\epsilon \mathbf{h})}^{\mu \nu}\right)_{, \rho}(\mathbf{X})\right) d S_{\mu}(\mathbf{X})
\end{aligned}
$$

(setting $U^{\mu}:=\sqrt{-g} V^{\mu}$ and with $\mathbf{g}_{(\epsilon \mathbf{h})}:=\mathbf{g}+\epsilon \mathbf{h}$ and $d S_{\mu}:=$ $\left.\varepsilon_{\mu \nu \rho \sigma} d x^{\nu} \wedge d x^{\rho} \wedge d \mathrm{x}^{\sigma}\right)$ when this integral makes sense, which is true if $\mathbf{g} \in \mathrm{E}^{\star}, \mathbf{h} \in \mathrm{E}$, and $|\epsilon|<a=a(\mathbf{g}, \mathbf{h})$. If, moreover, $\mathbf{h}(X)=0$ on $\partial \mathrm{U}$, we have $\mathbf{g}_{(\epsilon \mathbf{h})}(X)=\mathbf{g}(X)$ for any $X \in \partial \mathrm{U}$, so that integral (75) does not depend on $\epsilon \epsilon]-a, a$ [. Therefore, the LHS of (65) is zero. Since we have shown that this equation determines uniquely the functions $\mathbf{X} \mapsto T_{\mu \nu}(\mathbf{X}), \chi(\mathrm{K}) \rightarrow \mathbb{R}$, these functions are zero.

Let us finally prove the actual tensoriality of the "Hilbert tensor," whose components are defined by (53). Considering now any two charts $\chi$ and $\chi^{\prime}$ whose domain contains $\mathrm{K},(65)$ is true for any tensor field $\mathbf{h} \in \mathrm{E}$ that vanishes on $\partial \mathrm{U}$, using either $\chi$ or $\chi^{\prime}$ on the RHS (with primes for $\chi^{\prime}$ ). Since the LHS of (65) is invariant as is the action, so is the RHS; that is, we have for any such $\mathbf{h}$

$$
\int_{\Omega} T_{\mu \nu} h^{\mu \nu} \sqrt{-g} d^{4} \mathbf{X}=\int_{\Omega^{\prime}} T_{\mu \nu}^{\prime} h^{\prime \mu \nu} \sqrt{-g^{\prime}} d^{4} \mathbf{X}^{\prime} .
$$


Composing with the reverse coordinate maps $\chi^{-1}$ and $\chi^{\prime-1}$, we may regard $T_{\mu \nu}, h^{\mu \nu}, T_{\mu \nu}^{\prime}, h^{\prime \mu \nu}$ as functions defined over $\mathrm{K}=\chi^{-1}(\chi(\mathrm{K}))=\chi^{\prime-1}\left(\chi^{\prime}(\mathrm{K})\right)$, and we have for any such $\mathbf{h}$

$$
\int_{\mathrm{U}} T_{\mu \nu} h^{\mu \nu} d V_{4}=\int_{\mathrm{U}} T_{\mu \nu}^{\prime} h^{\prime \mu \nu} d V_{4}
$$

For any given tensor field $\mathbf{h}_{0} \in \mathrm{E}$, set $f:=T_{\mu \nu} h_{0}^{\mu \nu}$ and $f^{\prime}:=T_{\mu \nu}^{\prime} h_{0}^{\prime \mu \nu}$, which are thus two continuous functions on K. Consider any open domain W with $\mathrm{W} \subset \mathrm{U}$. Take any function $\phi$ which is defined and continuous over $\mathrm{K}$, which is $\mathscr{C}^{1}$ over $\mathrm{U}$, and that has compact support $\mathrm{K}^{\prime} \subset \mathrm{W}$. Define $\mathbf{h}:=\phi \mathbf{h}_{0}$. This is a tensor field that belongs to $\mathrm{E}$ and vanishes on $\partial \mathrm{W}$. Therefore, with the open domain $\mathrm{U}$ being arbitrary in the already proved point (ii) and hence in (77), we can apply (77) with $\mathrm{W}$ instead of $\mathrm{U}$. We thus get

$$
\int_{\mathrm{W}} f \phi d V_{4}=\int_{\mathrm{W}} f^{\prime} \phi d V_{4}
$$

Because this is true for any such function $\phi$, it follows that we have $f=f^{\prime}$ almost everywhere in W. Since these are continuous functions, we have $f=f^{\prime}$ in W. And since this is true for any open domain $\mathrm{W}$ with $\mathrm{W} \subset \mathrm{U}$, we have $f=f^{\prime}$ in $\mathrm{U}$ and hence also in $\mathrm{K}=\overline{\mathrm{U}}$. That is, $T_{\mu \nu} h_{0}^{\mu \nu}$ is invariant under coordinate changes, for whatever tensor field $\mathbf{h}_{0} \in \mathrm{E}$. Considering a given point $X \in \mathrm{K}$, we define a linear form $\Phi$ on the vector space $\mathscr{T}_{0}^{2}$ of the (2 0$)$ tensors at $X$, by setting

$$
\Phi(\underline{\mathbf{h}}):=T_{\mu \nu}(X) \underline{h}^{\mu \nu}, \quad \forall \underline{\mathbf{h}} \in \mathscr{T}_{0}^{2},
$$

which is thus independent of the chart. But the dual space of $\mathscr{T}_{0}^{2}$ is known (and easily checked) to be the vector space $\mathscr{T}_{2}^{0}$ of the $\left(\begin{array}{ll}0 & 2\end{array}\right)$ tensors $\underline{\mathbf{S}}$ at $X$. Hence, there is a unique tensor $\underline{\mathbf{S}} \in \mathscr{T}_{2}^{0}$ for which, in any chart, we have

$$
\Phi(\underline{\mathbf{h}})=S_{\mu \nu} \underline{h}^{\mu \nu}, \quad \forall \underline{\mathbf{h}} \in \mathscr{T}_{0}^{2} .
$$

From (79) and (80), it follows that, in any chart, the numbers $T_{\mu \nu}(X)$ are the components of the unique tensor $\underline{\mathbf{S}} \in \mathscr{T}_{2}^{0}$.

\section{A Uniqueness Result for the Energy Balance}

5.1. Is the Energy Balance Equation Unique? We begin with a discussion of this question for a system of isentropically deformable media in Newtonian gravity (NG). The energy balance (11) established in Section 2 for the matter field equations of NG has the form

$$
\partial_{\mu} V^{\mu}=\text { field source }:=-\rho \frac{\partial U}{\partial t},
$$

with the four-component column vector $\left(V^{\mu}\right)$ being here the "matter current" made with the matter energy density and flux:

$$
\left(V^{\mu}\right)=\left(w_{m}, \Phi_{m}\right)
$$

As we saw, (81) (i.e., (11)) is verified as soon as the following three equations are verified among the matter field equations: Newton's second law (1), the isentropy equation (5), and the continuity equation (9). For instance, we did not use the "constitutive equation" that relates the stress tensor to some deformation tensor or (for a barotropic fluid) that relates the pressure with the density. We note that, in view of (12) and (13), the matter current $\left(V^{\mu}\right)$ is polynomial in the local values of the fields $\phi^{A}(A=1, \ldots, n)=(\rho, \mathbf{v}, \Pi, \boldsymbol{\sigma}, U)$ that appear in those equations (thus, assigning in this sectioncontrary to Section 4-a different number $A$ to different components of a given vector or tensor field: $n=12$ here; the gravitational potential $U$ plays the same role as does the metric tensor in a Lagrangian for the matter fields in curved space-time, as was the case in the foregoing section). Now we ask if we can find a different expression for the matter current, say $V^{\prime \mu}$, for which the LHS of (81) would be always the same as with current (82), so that the same balance equation (81) would be valid with $V^{\prime \mu}$, when it is with $V^{\mu}$. Thus, can we change the matter current $V^{\mu}$ for another one $V^{\prime \mu}=V^{\mu}+W^{\mu}$, also polynomial with respect to the local values of the fields at any space-time point $X, q^{A}=\phi^{A}(X)$, so that the LHS of (81) would be unchanged for whatever values of the fields? That is, can we find a column four-vector $W^{\mu}$ which would be polynomial in $q^{A}$, s, such that we would have $\partial_{\mu} W^{\mu} \equiv 0$ ?

5.2. A Uniqueness Result. Thus, let $W^{\mu}$ be an order- $N$ polynomial in the field values $q^{A}=\phi^{A}(X)$, its coefficients being allowed to depend on the space-time position $X$ :

$$
\begin{aligned}
W^{\mu}\left(X, q^{A}\right)= & C_{0}^{\mu}(X)+C_{1 A}^{\mu}(X) q^{A}+\cdots \\
& +C_{N A_{1} \cdots A_{N}}^{\mu}(X) q^{A_{1}} \cdots q^{A_{N}} \\
& \left(A_{1} \leq \cdots \leq A_{N}\right) .
\end{aligned}
$$

Assume that its 4-divergence vanishes identically, $\partial_{\mu} W^{\mu} \equiv 0$ :

$$
\begin{aligned}
0 \equiv & C_{0, \mu}^{\mu}+C_{1 A, \mu}^{\mu} q^{A}+C_{1 A}^{\mu} q_{\mu}^{A}+\cdots \\
& +C_{N A_{1} \cdots A_{N}, \mu}^{\mu} q^{A_{1}} \ldots q^{A_{N}} \\
& +C_{N A_{1} \cdots A_{N}}^{\mu}\left(q_{\mu}^{A_{1}} q^{A_{2}} \ldots q^{A_{N}}+\cdots\right. \\
& \left.+q^{A_{1}} \ldots q^{A_{N-1}} q_{\mu}^{A_{N}}\right) \quad\left(q_{\mu}^{A}:=\phi_{, \mu}^{A}(X)\right) .
\end{aligned}
$$

That is, at any space-time point $X_{0}\left(x^{\rho}\right)$, (84) is valid for whatever possible values $q^{A}$ and $q_{\mu}^{A}$ of the fields and their derivatives at $X_{0}$. But, for whatever values $q^{A}$ and $q_{\mu}^{A}$ of these variables, there exist smooth functions $X \mapsto \phi^{A}(X)(A=$ $1, \ldots, n)$, defined in some neighborhood $U$ of $X_{0}$, such that we have

$$
\begin{aligned}
\phi^{A}\left(X_{0}\right) & =q^{A} \quad(A=1, \ldots, n), \\
\frac{\partial \phi^{A}}{\partial x^{\mu}}\left(X_{0}\right) & =q_{\mu}^{A} \quad(A=1, \ldots, n ; \mu=0, \ldots, 3) .
\end{aligned}
$$

Thus, our assumption means that on the RHS of (84) the polynomial function in the real variables $q^{A}$ and $q_{\mu}^{A}(A=$ 
$1, \ldots, n ; \mu=0, \ldots, 3)$ is identically zero. Hence, its coefficients are all zero. In particular,

$$
C_{1 A}^{\mu}\left(X_{0}\right)=0, \ldots, C_{N A_{1} \cdots A_{N}}^{\mu}\left(X_{0}\right)=0
$$

Thus, all coefficients in (83) are zero-except perhaps $C_{0}^{\mu}$, with $C_{0, \mu}^{\mu}=0$.

We thus got that we cannot alter the analytical expression of $w_{m}$ and $\Phi_{m}$ on the LHS of the matter energy balance (11) (apart from arbitrarily adding a zero-divergence vector field $C_{0}^{\mu}$ that is independent of the matter fields-this is indeed obviously possible, but we can get rid of this by asking that the matter current $\left(V^{\mu}\right)$ be polynomial in the fields and have no zero-order term, as is indeed the case in all concrete examples). The gravitational energy balance (14) has just the same form:

$$
\partial_{\mu} V^{\mu}=\text { matter source }:=\rho \frac{\partial U}{\partial t},
$$

where $V^{\mu}=\left(w_{g}, \Phi_{g}\right)$ is polynomial in the gravitational field $\phi^{A}(A=1, \ldots, 4)=\left(\partial_{\mu} U\right)$. It is valid when the gravitational field equation is. Therefore, similarly, as we found for the matter field energy balance, we cannot alter the analytical expression (14) of the gravitational energy balance.

5.3. Generalization. These results are clearly general. Consider, for example, the Maxwell electromagnetic field instead of the Newtonian gravitational field. The energy balance of the e.m. field is

$$
\frac{\partial w_{\mathrm{em}}}{\partial t}+\operatorname{div} \boldsymbol{\Phi}_{\mathrm{em}}=-\mathbf{j} \cdot \mathbf{E}
$$

with $w_{\mathrm{em}}:=\left(\mathbf{E}^{2}+\mathbf{B}^{2}\right) / 8 \pi$ the volume energy density of the electromagnetic field and $\Phi_{\mathrm{em}}:=(\mathbf{E} \wedge \mathrm{B}) / 4 \pi$ the electromagnetic energy flux. The same uniqueness result says that we cannot find an alternative expression for $w_{\mathrm{em}}$ and $\Phi_{\mathrm{em}}$ on the LHS, which would be valid for whatever values of the fields $\mathbf{E}$ and $\mathbf{B}$ and their first derivatives.

\section{Conclusion}

The classical concept of energy emerges from an analysis of the power done, first in the case of a mass point and then for the case of a volume element in a continuous medium. We have argued that, in the case of a continuous medium or a system of fields, the meaning of the energy conservation is primarily local: it says that, in any bounded domain, the energy loss or gain is due only to a well-identified flux that goes through the boundary of that domain. Thus, it expresses in a general way the Lavoisier principle: "Nothing is lost, nothing is created, everything transforms." While it is of course interesting also and even often important to have global energy conservation laws, this interest is limited by two facts: (i) an exact global conservation law can be hoped, strictly speaking, only for the Universe as a whole, because there are energy exchanges at all scales-but physics can not be reduced to cosmology. (ii) A global energy conservation law says merely that one number is a constant: the total energy; in the most favorable case with global conservation of the energy, the momentum, and the angular momentum, ten numbers are constant. In the relevant case of a system of fields, however, there are an infinite number of degrees of freedom, so this is only a small part of the information needed.

We have tried to precisely state and prove the main results regarding the derivation of the Hilbert tensor from the invariance of the action in generally covariant theories. We hope to have proved these results in a convincing way, keeping the mathematical sophistication to the minimum needed. The Hilbert tensor theory is beautiful and is essential to general relativity. It is important also in relativistic quantum mechanics. One should note, however, that historically the main examples of the energy-momentum tensor have been derived from the corresponding local conservation equations for energy and momentum [24], whence the interest in examining the uniqueness of the latter kind of equations.

\section{Appendix}

\section{Regular Domains}

Definition A.1 (see [25]). Let $\mathrm{M}$ be a differentiable manifold, with dimension $d$. One will call closed domain of $M$ any closed subset $\mathrm{D}$ of $\mathrm{M}$ such that, for any $x \in \mathrm{D}$, either (i) there is an open subset $\mathrm{W}$ of $\mathrm{M}$ such that $x \in \mathrm{W} \subset \mathrm{D}$ or (ii) there is a chart $(\mathrm{W}, \varphi)$ with $\varphi(x)=\mathbf{0}$ and $\varphi(y)=\left(y^{1}, \ldots, y^{d}\right)$ for $y \in W$, such that

$$
\begin{aligned}
\mathrm{W} \cap \mathrm{D} & =\left\{y \in \mathrm{W} ; y^{1} \leq 0\right\}, \\
\text { i.e., } \varphi(\mathrm{W} \cap \mathrm{D}) & =\left\{\mathrm{y} \in \varphi(\mathrm{W}) ; y^{1} \leq 0\right\} .
\end{aligned}
$$

Proposition A.2 (see [25]). Let D be a closed domain of a $d$ dimensional differentiable manifold $\mathrm{M}$. In case (i), the point $x$ is in $\mathrm{D}^{\mathrm{D}}$, the interior of $\mathrm{D}$ (i.e., the largest open set $\mathrm{U}$ of $\mathrm{M}$, such that $\mathrm{U} \subset \mathrm{D})$. In case (ii), the point $x$ is in $\partial \mathrm{D}$, the boundary of $\mathrm{D}$, which is $a(d-1)$-dimensional submanifold of the differentiable manifold $\mathrm{M}$.

Recall that the (topological) boundary of any subset $\mathrm{A}$ of $\mathrm{M}$ is defined to be $\partial \mathrm{A}:=\overline{\mathrm{A}} \cap \overline{\mathrm{CA}}$, where the overbar means the adherence (or closure) in $\mathrm{M}$ and CA means the complementary set of A in M. It is easy to prove (cf. [26]) that we have always

$$
\begin{aligned}
& \AA \cap \partial \mathrm{A}=\emptyset, \\
& \AA \cup \partial \mathrm{A}=\overline{\mathrm{A}} .
\end{aligned}
$$

If $\mathrm{M}$ is an oriented manifold, then the Stokes theorem (and thus also the divergence theorem) applies to any differential $(d-1)$-form (resp., to any continuously differentiable vector field), in any closed domain $\mathrm{D}$ of $\mathrm{M}$ with its boundary $\partial \mathrm{D}$ [25]. 
Proposition A.3. Let D be a closed domain (see Definition A.1) of a d-dimensional differentiable manifold $\mathrm{M}$ and let $\mathrm{U}:=\stackrel{\mathrm{D}}{\mathrm{D}}$ be its interior. One has

$$
\overline{\mathrm{U}}=\mathrm{D} ;
$$

that is, a closed domain is the closure of its interior. We thus call $\mathrm{U}:=\stackrel{\mathrm{D}}{\mathrm{D}}$ an open domain of M. Moreover, one has

$$
\overline{\mathrm{CD}}=\mathrm{CU} \text {. }
$$

Proof. Since D is a closed set such that $U \subset D$, we have $\bar{U} \subset D$. Due to (A.3), in order to prove that $\mathrm{D} \subset \overline{\mathrm{U}}$, we just have to prove that $\partial \mathrm{D} \subset \overline{\mathrm{U}}$. If $x \in \partial \mathrm{D}$, we may apply to it case (ii) of Definition A.1. Let A be any open neighborhood of $x$. We will show that it intersects both U and CD; to show this, we may assume that $\mathrm{A} \subset \mathrm{W}$, with $\mathrm{W}$ being the domain of the chart $\varphi$. Thus, $\varphi(\mathrm{A})$ is an open neighborhood of $\varphi(x)=\mathbf{0}$ in $\mathbb{R}^{d}$; hence, it contains a ball $\left|y^{j}\right|<r(j=1, \ldots, d)$. (a) Take first $\mathbf{y}_{0}=\left(y^{j}\right)$ such that $-r<y^{1}<0$ and $y^{j}=0$ for $j=2, \ldots, d$; then $\mathbf{y}_{0} \in \varphi(\mathrm{A}) \subset \varphi(\mathrm{W})$. But we get from (A.1) that any point $\mathbf{y} \in \varphi(\mathrm{W})$ such that $y^{1}<0$ is in the interior of $\varphi(\mathrm{D} \cap \mathrm{W})$. Thus, $y_{0} \in \varphi(A)$ is in the interior of $\varphi(D \cap W)$, or equivalently $y_{0}=\varphi^{-1}\left(\mathbf{y}_{0}\right) \in \mathrm{A}$ is in the interior of $\mathrm{D} \cap \mathrm{W}$, hence in $\mathrm{U}=\stackrel{\mathrm{D}}{\text {. }}$. So, $\partial \mathrm{D} \subset \overline{\mathrm{U}}$; hence, (A.4) is proved. (b) On the other hand, take now $\mathbf{y}_{1}=\left(y^{j}\right)$ such that $0<y^{1}<r$ and $y^{j}=0$ for $j=$ $2, \ldots, d$ : also $\mathbf{y}_{1} \in \varphi(\mathrm{A}) \subset \varphi(\mathrm{W})$, but we get from (A.1) that $y_{1}=\varphi^{-1}\left(\mathbf{y}_{1}\right) \in \mathrm{A}$ is in CD. Thus, we have also $\partial \mathrm{D} \subset \overline{\mathrm{CD}}$, whence $\overline{\mathrm{CD}} \cup \partial \mathrm{D}=\overline{\mathrm{CD}}$. But, from (A.2) and (A.3), we have $\mathrm{D} \cap \mathrm{C} \partial \mathrm{D}=\mathrm{U}$, or $(\mathrm{CD}) \cup \partial \mathrm{D}=\mathrm{C} U$. Therefore,

$$
\mathrm{CU}=\overline{\mathrm{CU}}=\overline{\mathrm{CD}} \cup \partial \mathrm{D}=\overline{\mathrm{CD}},
$$

which proves (A.5).

Corollary A.4. Let $\mathrm{U}$ be an open domain of $\mathrm{M}$ and let $f$ be a continuous real function defined in a neighborhood of $\mathrm{D}=\overline{\mathrm{U}}$. In order that Supp $f \subset \mathrm{D}$, it is necessary and sufficient that $f(x)=0$ if $x \notin \mathrm{U}$.

Proof. The support of $f$, Supp $f$, is defined to be the smallest closed set containing the set of the points $x$ such that $f(x) \neq$ 0 , or equivalently $C$ Supp $f$ is the largest open set $\Omega$ such that $f_{\mid \Omega}=0$. Therefore, with CD being an open set,

$$
(\operatorname{Supp} f \subset \mathrm{D}) \Longleftrightarrow\left(f_{\mathrm{ICD}}=0\right) \text {. }
$$

Since $f$ is continuous, $f_{\mid C D}=0$ is equivalent to $f_{\mid \overline{C D}}=0$-that is, from (A.5), to $f_{\mid C U}=0$.

\section{Competing Interests}

The author declares that there are no competing interests regarding the publication of this paper.

\section{Acknowledgments}

The author is grateful to T. Padmanabhan for pointing out [22] to him.

\section{Endnotes}

1. Of course, $\nabla U$ is the spatial vector having components $(\nabla U)^{i}=U_{, i}(i=1,2,3)$ in any Cartesian coordinate system ("Cartesian components"), that is, in any coordinate system for which we have the identity $h_{i j} \equiv \delta_{i j}$, where $\mathbf{h}$ is the (Euclidean) spatial metric. Also, $\operatorname{div} \boldsymbol{\sigma}$ is the spatial vector with Cartesian components $(\operatorname{div} \boldsymbol{\sigma})^{i}=$ $\sigma^{i j}{ }_{j}$. And $\operatorname{grad} \mathbf{v}$ is the mixed spatial tensor having Cartesian components $(\operatorname{grad} \mathbf{v})^{i}{ }_{j}=v^{i}{ }_{j}$. Mixed tensors are identified with linear mappings: $(\operatorname{grad} \mathbf{v})(\mathbf{w})$ is the spatial vector having components $(\operatorname{grad} \mathbf{v}){ }_{j}^{i} w^{j}$, which is the same vector independently of the coordinate system. We use the spatial metric $\mathbf{h}$ to raise or lower the indices; for example, $v_{i}:=h_{i j} v^{j}$. The equations in this section are valid in any spatial coordinates, whether Cartesian or curvilinear.

2. $\sigma(\mathbf{v})$ is the vector transformed of $\mathbf{v}$ by the linear mapping associated with the mixed tensor form of $\boldsymbol{\sigma}$; thus, $[\boldsymbol{\sigma}(\mathbf{v})]^{i}:=\sigma^{i}{ }_{j} v^{j}=\sigma^{i j} v_{j}$. And $\boldsymbol{\sigma}: \mathbf{D}:=\sigma^{i}{ }_{j} D^{j}{ }_{i}$ is the scalar product of mixed tensors, defined by double contraction.

3. If $\phi^{A}$ is a section of a vector bundle with base $V$, say $E$, to write its local expression needs that not only a chart on $\mathrm{V}$ but also a frame field $\left(e_{a}\right)$ on $\mathrm{E}$ is given. However, in the case of a tensor field, a relevant frame field is determined uniquely by the data of the chart $\chi$ with the associated natural basis $\left(\partial_{\mu}\right)$ and the dual basis $\left(d x^{\mu}\right)$. In a very general case, $\mathrm{E}$ has the form $\mathrm{E}=\mathrm{T} \otimes \mathrm{N}$ with $\mathrm{T}$ being an usual tensor bundle on $\mathrm{V}$ and $\mathrm{N}$ being a vector bundle of a different kind. Then, it is natural to take a frame field of the form $\left(T_{b} \otimes N_{c}\right)$, with $\left(T_{b}\right)$ being a frame field on $\mathrm{T}$, determined by the chart $\chi$, and with $\left(N_{c}\right)$ being a frame field on $\mathrm{N}$, which is left unchanged when changing the chart. Thus, the transformation law on changing the chart is determined.

4. Considering "every compact subset $\mathrm{K}$ of $\mathrm{V}$ " [8] is too general, because one needs to use the divergence theorem on $\mathrm{K}$ to eliminate a surface term, as is done below to get (44) and (52) and as is done for the equation after Eq. (222) in [8]. One should assume that the compact set is a "closed domain." A closed domain D is the closure of its interior $\stackrel{\circ}{\mathrm{D}}$ (Proposition A.3); hence, it is equivalent to start from a bounded open domain $\mathrm{U}$ and to define $\mathrm{K}=\overline{\mathrm{U}}$, which is a compact domain, or to start from $\mathrm{K}$ and to define $\mathrm{U}=\stackrel{\circ}{\mathrm{K}}$. Then, of course, the smooth boundary $\partial \mathrm{U}$ has measure zero with respect to the invariant four-dimensional measure $\sqrt{-g} d^{4} \mathbf{X}$. Hence, the action integral (31), as well as its variation (52) or (54), is unaltered if instead of $U$ we consider its closure $\mathrm{K}$ as the integration domain, as considered in [8]. Thus, when the compact $\mathrm{K}$ considered in [8] is a closed domain, as it should be imposed, the definition of the action $S_{\mathrm{K}}$ and the condition imposed on the vector field $X$ in [8] are equivalent to those considered here for $S_{U}$ and $\xi$. 
5. In [2], it is "set $\delta g^{\mu \nu}=0$ at the integration limits" and, later on, it is stated that " $\xi^{\mu}$ vanish at the limits of the integration," without any explanation or any link between the two statements. Note that assuming merely that $\xi^{\mu}=0$ on the boundary $\partial \mathrm{U}$ does not imply that $\delta g^{\mu \nu}=0$ on $\partial \mathrm{U}$.

6. All sixteen $\underline{g}^{\mu \nu}$ 's $(0 \leq \mu \leq 3,0 \leq \nu \leq 3)$ are considered as independent variables in $\mathscr{L}$ for the calculation of $\partial \mathscr{L} / \partial \underline{g}^{\mu \nu}$, even though $g^{\mu \nu}=g^{\nu \mu}$ : see Note $\ddagger$ on p. 269 in [2]. Thus, all sixty-four $\underline{g}_{\rho}^{\mu \nu}$ s $(0 \leq \mu \leq 3,0 \leq \nu \leq 3,0 \leq$ $\rho \leq 3$ ) are also considered as independent variables for the calculation of $\partial \mathscr{L} / \partial \underline{g}_{\rho}^{\mu \nu}$.

7. Recalling Note 6 , the symmetry of the metric does not by itself imply the symmetry of components (53): check, for example, $L=\left(g^{12}\right)^{2}$, which of course is not invariant.

8. Actually, in the theory [21], the energy density and its flux have been defined only in the preferred reference frame assumed by the theory.

\section{References}

[1] I. Müller and W. H. Müller, Fundamentals of Thermodynamics and Applications, Springer, Berlin, Germany, 2009.

[2] E. M. Lifshitz and L. D. Landau, The Classical Theory of Fields, Pergamon, Oxford, UK, 3rd edition, 1971.

[3] V. Fock, The Theory of Space, Time and Gravitation, Pergamon, Oxford, UK, 1st edition, 1959.

[4] F. J. Belinfante, "On the spin angular momentum of mesons," Physica, vol. 6, pp. 887-898, 1939.

[5] A. Trautman, "On the conservation theorems and equations of motion in covariant field theories," Bulletin de l'Académie Polonaise des Sciences, Cl. 3, vol. 4, no. 10, pp. 675-678, 1956.

[6] R. M. Wald, General Relativity, University of Chicago Press, Chicago, Ill, USA, 1984.

[7] M. J. Gotay and J. E. Marsden, "Stress-energy-momentum tensors and the Belinfante-Rosenfeld formula," in Mathematical Aspects of Classical Field Theory, vol. 132 of Contemporary Mathematics, pp. 367-392, 1992.

[8] M. Forger and H. Römer, "Currents and the energy-momentum tensor in classical field theory: a fresh look at an old problem," Annals of Physics, vol. 309, no. 2, pp. 306-389, 2004.

[9] M. Leclerc, "Canonical and gravitational stress-energy tensors," International Journal of Modern Physics D, vol. 15, no. 7, pp. 959989, 2006.

[10] M. Arminjon, "Energy and equations of motion in a tentative theory of gravity with a privileged reference frame," Archives of Mechanics, vol. 48, no. 1, pp. 25-52, 1996.

[11] S. Chandrasekhar, "Conservation laws in general relativity and in the post-Newtonian approximations," The Astrophysical Journal, vol. 158, pp. 45-54, 1969.

[12] M. Arminjon and F. Reifler, "General reference frames and their associated space manifolds," International Journal of Geometric Methods in Modern Physics, vol. 8, no. 1, pp. 155-165, 2011.

[13] M. Arminjon, "Some remarks on quantum mechanics in a curved spacetime, especially for a Dirac particle," International Journal of Theoretical Physics, vol. 54, no. 7, pp. 2218-2235, 2015.

[14] P. J. Olver, "Lectures on Lie groups and differential equations," in Symmetries of Variational Problems, chapter 5, 2012.
[15] M. Arminjon, "A simpler solution of the non-uniqueness problem of the covariant Dirac theory," International Journal of Geometric Methods in Modern Physics, vol. 10, no. 7, Article ID 1350027, 24 pages, 2013.

[16] B. A. Dubrovin, A. T. Fomenko, and S. P. Novikov, Modern Geometry-Methods and Applications, Part I, Springer, New York, NY, USA, 2nd edition, 1992.

[17] H. Stephani, General Relativity-An Introduction to the Theory of the Gravitational Field, English Edition, Cambridge University Press, Cambridge, UK, 1982.

[18] N. Rosen, "Flat-space metric in general relativity theory," Annals of Physics, vol. 22, pp. 1-11, 1963.

[19] A. A. Logunov and M. A. Mestvirishvili, The Relativistic Theory of Gravitation, Mir Publishers, Moscow, Russia, 1989.

[20] W. Petry, "Gravitation in flat space-time and general relativity," Journal of Applied Mathematics and Physics, vol. 2, pp. 50-54, 2014.

[21] M. Arminjon, "Space isotropy and weak equivalence principle in a scalar theory of gravity," Brazilian Journal of Physics, vol. 36, no. 1, pp. 177-189, 2006.

[22] T. Padmanabhan, "Momentum density of spacetime and the gravitational dynamics," General Relativity and Gravitation, vol. 48, article 4, 2016.

[23] C. Cattaneo, "General relativity: relative standard mass, momentum, energy and gravitational field in a general system of reference," Nuovo Cimento, vol. 10, pp. 318-337, 1958.

[24] J.-P. Provost, "A brief history of the energy-momentum tensor; 1900-1912," in Proceedings of the 13th Marcel Grossmann Meeting, R. T. Jantzen, K. Rosquist, and R. Ruffini, Eds., pp. 20542056, World Scientific, Stockholm, Sweden, July 2012.

[25] M. Berger and B. Gostiaux, Differential Geometry: Manifolds, Curves, and Surfaces, Springer, New York, NY, USA, 1988.

[26] J. Dieudonné, Treatise on Analysis, Volume 1: Foundations of Modern Analysis, Academic Press, New York, NY, USA, 1969. 


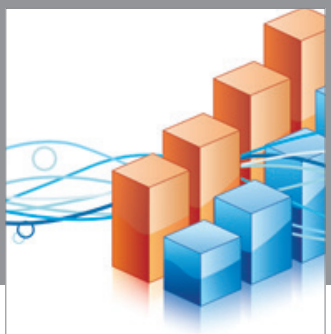

Advances in

Operations Research

vatem alat4

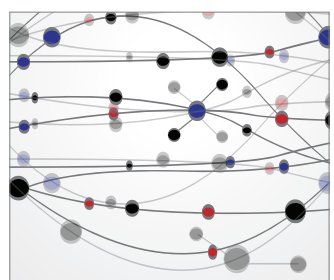

\section{The Scientific} World Journal
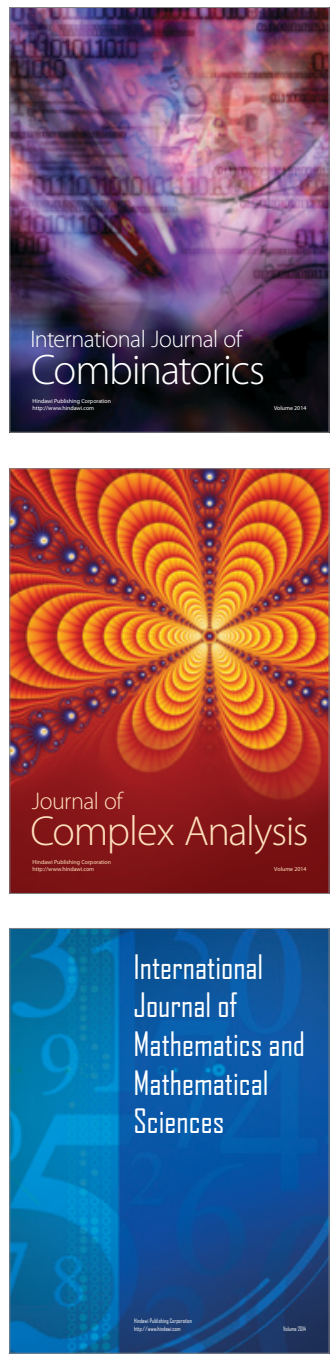
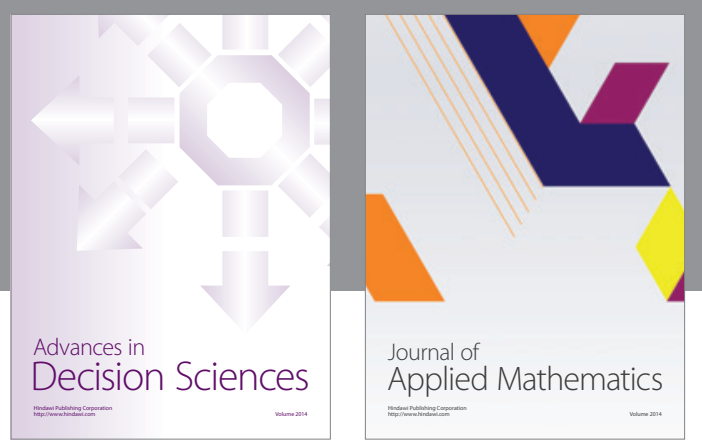

Algebra

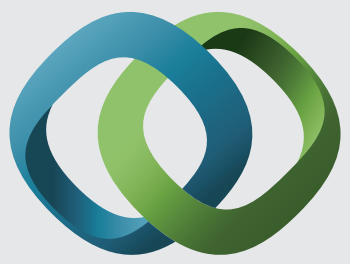

\section{Hindawi}

Submit your manuscripts at

http://www.hindawi.com
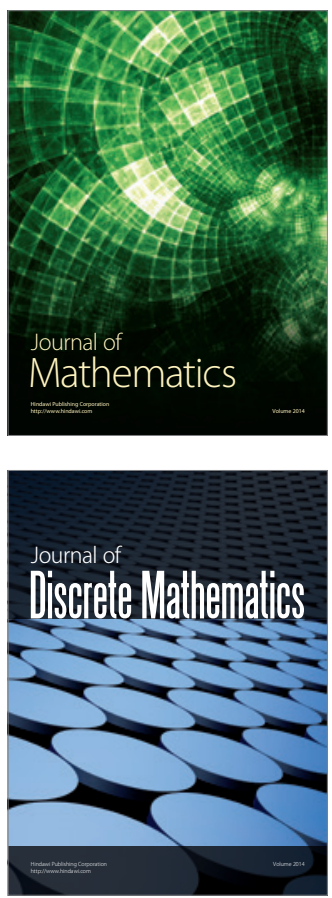

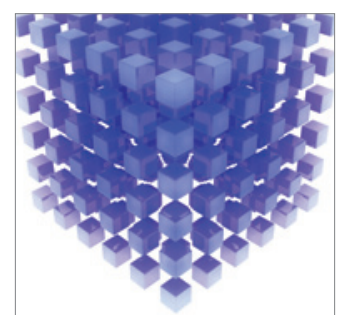

Mathematical Problems in Engineering
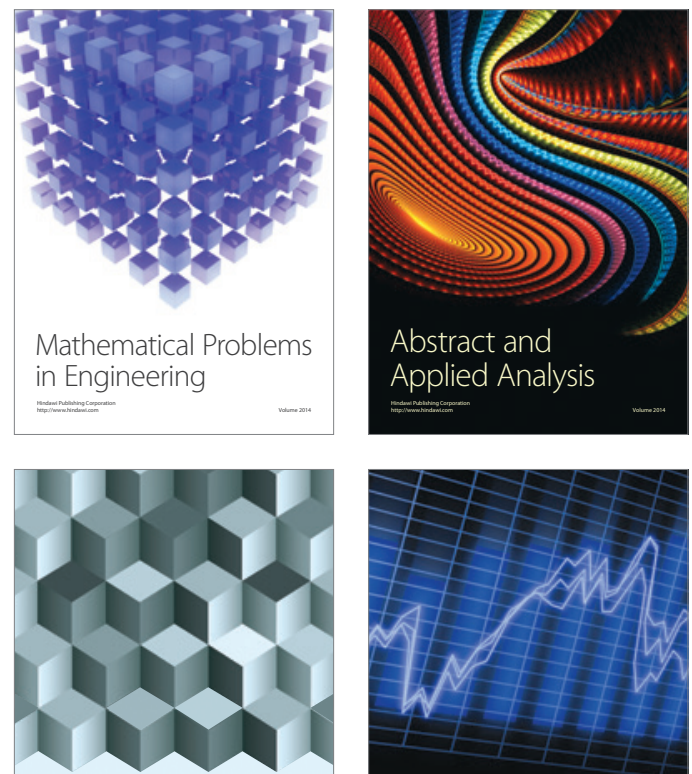

Journal of

Function Spaces

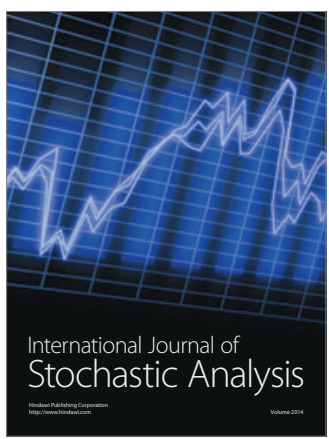

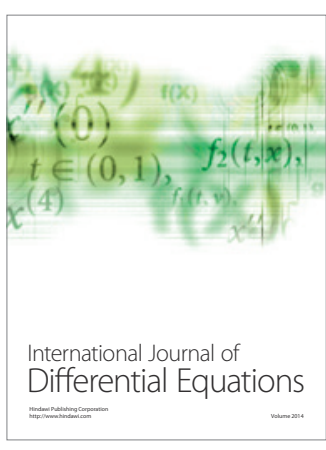
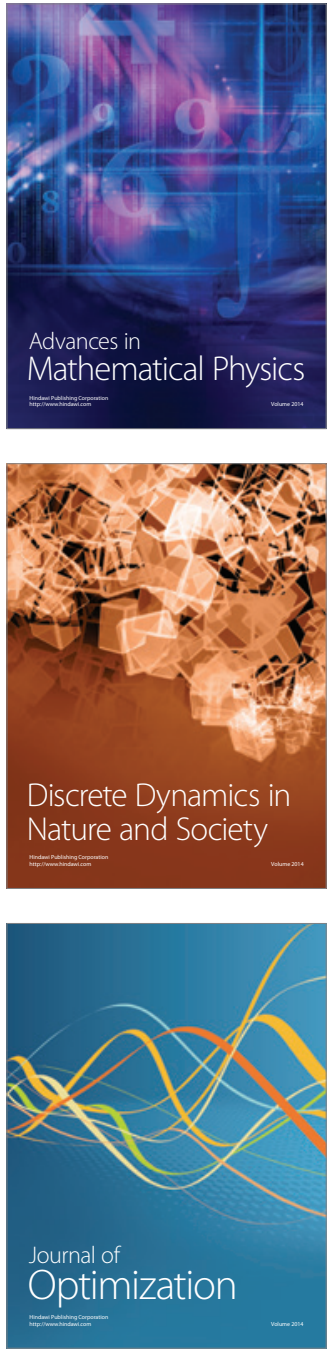